\title{
Recent Developments in Few-Nucleon Scattering
}

\author{
A. Kievsky ${ }^{\mathrm{a}}$ \\ Istituto Nazionale di Fisica Nucleare, Largo B. Pontecorvo 3, 56127 (Pisa), Italy
}

\begin{abstract}
Using modern nucleon-nucleon interactions in the description of the $A=3,4$ nuclei, it is not possible to reproduce both the three- and four-nucleon binding energies simultaneously. This is one manifestation of the necessity of including a three-nucleon force in the nuclear Hamiltonian. Several models of the three-nucleon force exist and are applied in the description of light nuclei. However, as it is discussed here, a simultaneous description of the three- and four-body binding energies and the $n-d$ doublet scattering length seems to be problematic. Accordingly, a comparative study of some of these models is performed. In a different analysis, we study applications of the Kohn Variational Principle, formulated in terms of integral relations, to describe $N-d$ scattering processes.
\end{abstract}

\section{Introduction}

Realistic nucleon-nucleon $(\mathrm{NN})$ potentials reproduce the experimental $\mathrm{NN}$ scattering data up to energies of $350 \mathrm{MeV}$ with a $\chi^{2}$ per datum close to 1 . However, the use of these potentials in the description of the three- and four-nucleon bound and scattering states gives a $\chi^{2}$ per datum much larger than 1 (see for example Refs.[1,2]). In order to improve that situation, different three-nucleon force (TNF) models have been introduced so far. Widely used in the literature are the Tucson-Melbourne (TM) and the Urbana IX (URIX) models [3,4]. These models are based on the exchange mechanism of two pions between three nucleons with the intermediate excitation of a $\Delta$ resonance. The TM model has been revisited within a chiral symmetry approach [5], and it has been demonstrated that the contact term present in it should be dropped. This new TM potential, known as $\mathrm{TM}^{\prime}$, has been subsequently readjusted [6]. The final operator structure coincides with that one given in the TNF of Brazil already derived many years ago [7]. Recently, TNFs have been derived based on chiral effective field theory at next-to-next-to-leading order [8]. A local version of these interactions (hereafter referred as N2LOL) can be found in Ref. [9]. All these models contain a certain number of parameters that fix the strength of the interaction. It is a common practice to determine these parameters from the three- and four-nucleon binding energies. A particular TNF is in general associated to a specific NN potential and the sum of the two interactions forms the nuclear potential energy. The two- and three-nucleon interactions derived using chiral effective field theory are consistently constructed. However a particular TNF can be used associated with different NN interactions. As a consequence, the parametrization of a particular TNF could change since different NN potentials predict different $A=3,4$ binding energies.

\footnotetext{
${ }^{a}$ e-mail: kievsky@pi.infn.it
}

More recently, a new class of two-nucleon interactions has been obtained ( $V_{\text {low- } k}$ potentials). With the purpose of eliminating the high-momentum part of the interaction, the Hilbert space has been separated into low and high momentum regions and the renormalization group method has been used to integrate out the high momentum components above a cutoff $\Lambda$ [10]. The value for $\Lambda$ is typically chosen to reproduce the triton binding energy.

All these potential models can be used to study bound and scattering states in the $A=3,4$ systems in order to extract information about their capability to describe the nuclear dynamics. Besides the bound state energies, in the $A=3$ system, the $n-d$ doublet scattering length ${ }^{2} a_{n d}$ can give valuable information. In principle this quantity is correlated, to some extent, to the $A=3$ binding energy through the so-called Phillips line $[11,12]$. However the presence of TNFs of the type studied here breaks this correlation. Therefore ${ }^{2} a_{n d}$ emerges as an independent observable. Due to the lack of excited states in the $A=3$ system, the zero energy state is the first one above the ground state. In the case of $n-d$ scattering at zero energy, the $J=\frac{1}{2}^{+}$ state is orthogonal to the triton ground state and, for this reason, it presents a node in the relative distance between the incident nucleon and the deuteron. The position of the node is related to the scattering length and it is also sensitive to the relation between the overall attraction and repulsion of the interaction. Several of the realistic NN potentials underestimate the triton binding energy. Therefore by adding a TNF, with the strength fixed for example to reproduce the triton binding energy, the balance between the total attraction and repulsion in the potential changes. This leads to a modification in ${ }^{2} a_{n d}$ and this modification depends on the parameters in the TNF. The determination of the TNF parametrization able to describe the triton binding energy $B\left({ }^{3} \mathrm{H}\right)$, the $\alpha$-particle binding energy $B\left({ }^{4} \mathrm{He}\right)$ and ${ }^{2} a_{n d}$ has been analyzed in Ref. [8] for a TNF derived from 
chiral effective field theory. A similar analysis has not been done for the local TNF models URIX, TM' and N2LOL.

In Refs. [13,14] results for $B\left({ }^{3} \mathrm{H}\right), B\left({ }^{4} \mathrm{He}\right),{ }^{2} a_{n d}$ are given using different combinations of $\mathrm{NN}$ interactions (see Table 1). Those results indicate that the models are not able to describe simultaneously the $A=3,4$ binding energies and ${ }^{2} a_{n d}$. In order to analyze further the mentioned discrepancies, here we study potential models constructed summing to the AV18 NN potential [15] the three-nucleon interactions of TM', URIX and N2LOL. Parametrizations of the URIX and TM' models already exist in conjunction with the AV18 potential. Conversely the N2LOL force has been constructed using the N3LO-Idaho potential from Entem et al. [16]. So, here we adapt its parametrization to reproduce, when summed to the AV18 interaction, the triton binding energy. Different parametrizations of the three TNF models are analyzed studying the description of $B\left({ }^{3} \mathrm{H}\right)$, $B\left({ }^{4} \mathrm{He}\right)$ and ${ }^{2} a_{n d}$ and some polarization observables in $p-d$ scattering. The calculations have been done using the hyperspherical harmonic $(\mathrm{HH})$ method as given in Refs. [1720] to describe bound and scattering states in $A=3,4$ systems using local potentials. The extension to treat nonlocal potentials was given in Refs. [14,21].

In a different application devoted to study scattering states in few-nucleon systems, a discussion of the use of the integral relations derived in Ref. [22] from the Kohn Variational principle (KVP) is given. It has been shown that starting from the KVP, the tangent of the phase-shift can be put in a form of a quotient where both, the numerator and the denominator, are given in the form of an integral relation. This is similar to what was proposed in Ref. [23], however its strict relation with the KVP has not been recognized. To be noticed that a general formulation of the scattering theory using surface-integrals is given in Ref. [24]. Here we would like to discuss some specific examples of the integral relations derived from the KVP. Starting the analysis in the simplest case, the $A=2$ system, we show that they can be used to compute phase-shifts from bound state like functions. A second application of the integral relations regards the possibility of determining phase-shifts from a calculation in which the Coulomb potential has been screened. All these examples serve to demonstrate the general validity of the KVP formulated in terms of integral relations. Due to their short-range nature, they are determined by the wave function in the interaction region and not from its explicit asymptotic behaviour. This means that each wave function solving $(H-E) \Psi=0$ in the interaction region can be used to determine the corresponding scattering amplitude even if its asymptotic behaviour is not the physical one.

\section{The HH expansion for $A=3,4$ systems}

In this section we briefly review the $\mathrm{HH}$ method for bound and scattering states.

\subsection{The HH Method for Bound States}

The nuclear wave function for the three-body system can be written as

$$
|\Psi\rangle=\sum_{\mu} c_{\mu}\left|\Psi_{\mu}\right\rangle
$$

where $\left|\Psi_{\mu}\right\rangle$ is a suitable complete set of states, and $\mu$ is an index denoting the set of quantum numbers necessary to completely specify the basis elements.

The coefficients of the expansion can be calculated using the Rayleigh-Ritz variational principle, which states that

$$
\left\langle\delta_{c} \Psi|H-E| \Psi\right\rangle=0,
$$

where $\delta_{c} \Psi$ indicates the variation of $\Psi$ for arbitrary infinitesimal changes of the linear coefficients $c_{\mu}$. Where the Hamiltonian of the system consists in the kinetic part plus two- and three-nucleon interaction terms

$$
H=-\frac{\hbar^{2}}{2 m} \sum_{i} \nabla_{i}^{2}+\sum_{i<j} V(i, j)+\sum_{i<j<k} W(i, j, k)
$$

The problem of determining $c_{\mu}$ and the energy $E$ is reduced to a generalized eigenvalue problem,

$$
\sum_{\mu^{\prime}}\left\langle\Psi_{\mu}|H-E| \Psi_{\mu^{\prime}}\right\rangle c_{\mu^{\prime}}=0
$$

The main difficulty of the method is to compute the matrix elements of the Hamiltonian $H$ with respect to the basis states $\left|\Psi_{\mu}\right\rangle$. Usually $H$ is given as a sum of terms (kinetic energy, two-body potential, etc.). The calculation of the matrix elements of some parts of $H$ can be more conveniently performed in coordinate space, while for other parts it could be easier to work in momentum space. Therefore, it is important that the basis states $\left|\Psi_{\mu}\right\rangle$ have simple expressions in both spaces. The HH functions indeed have such a property.

In the case of three nucleons of mass $m$ the Jacobi vectors $\boldsymbol{x}_{1 p}, \boldsymbol{x}_{2 p}$ correspond to a given particle permutation denoted with $p$, which specifies the particle order $i, j, k$,

$$
\begin{aligned}
& \boldsymbol{x}_{2 p}=\frac{1}{\sqrt{2}}\left(\boldsymbol{r}_{j}-\boldsymbol{r}_{i}\right), \\
& \boldsymbol{x}_{1 p}=\sqrt{\frac{2}{3}}\left(\boldsymbol{r}_{k}-\frac{1}{2}\left(\boldsymbol{r}_{i}+\boldsymbol{r}_{j}\right)\right) .
\end{aligned}
$$

Here $p=1$ corresponds to the order 1,2,3. It is convenient to replace the modulii of $\boldsymbol{x}_{2 p}$ and $\boldsymbol{x}_{1 p}$ with the so-called hyperradius and hyperangle, defined as

$$
\begin{aligned}
\rho & =\sqrt{\boldsymbol{x}_{1 p}^{2}+\boldsymbol{x}_{2 p}^{2}}, \\
\tan \phi_{p} & =\frac{x_{1 p}}{x_{2 p}} .
\end{aligned}
$$

Note that $\rho$ does not depend on the particle permutation $p$. The complete set of hyperspherical coordinates is then given by $\left\{\rho, \Omega_{p}^{(\rho)}\right\}$, with

$$
\Omega_{p}^{(\rho)}=\left[\hat{\boldsymbol{x}}_{1 p}, \hat{\boldsymbol{x}}_{2 p} ; \phi_{p}\right],
$$




\section{$19^{\text {th }}$ International IUPAP Conference on Few-Body Problems in Physics}

and the suffix $(\rho)$ recalls the use of the coordinate space.

The expansion states $\left|\Psi_{\mu}\right\rangle$ of Eq. (1) are then given by

$$
\left|\Psi_{\mu}^{(\rho)}\right\rangle=f_{l}(\rho) \mathcal{Y}_{\{G\}}\left(\Omega^{(\rho)}\right),
$$

where $f_{l}(\rho)$ for $l=1, \ldots M$ is a complete set of hyperradial functions, chosen of the form

$$
f_{l}(\rho)=\gamma^{3} \sqrt{\frac{l !}{(l+5) !}} L_{l}^{(5)}(\gamma \rho) \mathrm{e}^{-\frac{\gamma}{2} \rho}
$$

Here $L_{l}^{(5)}(\gamma \rho)$ are Laguerre polynomials, and the non-linear parameter $\gamma$ is variationally optimized. As an example, for the N3LO-Idaho potential, it can be chosen in the interval $6-8 \mathrm{fm}^{-1}$.

The functions $\mathcal{Y}_{\{G\}}\left(\Omega^{(\rho)}\right)$ are written as

$$
\mathcal{Y}_{\{G\}}\left(\Omega^{(\rho)}\right)=\sum_{p=1}^{3}\left[Y_{[G]}^{L L_{z}}\left(\Omega_{p}^{(\rho)}\right) \otimes\left[S_{2} \otimes \frac{1}{2}\right]_{S S_{z}}\right]_{J J_{z}}\left[T_{2} \otimes \frac{1}{2}\right]_{T T_{z}},
$$

where the sum is performed over the three even permutations. The spin (isospin) of particles $i$ and $j$ are coupled to $S_{2}\left(T_{2}\right)$, which is itself coupled to the spin (isospin) of the third particle to give the state with total spin $S$ (isospin $T, T_{z}$ ). The total orbital angular momentum $L$ and the total spin $S$ are coupled to the total angular momentum $J, J_{z}$. The functions $Y_{[G]}^{L L_{z}}\left(\Omega_{p}^{(\rho)}\right)$, having a definite value of $L, L_{z}$, are the $\mathrm{HH}$ functions:

$$
Y_{[G]}^{L L_{z}}\left(\Omega_{p}^{(\rho)}\right)=\left[Y_{\ell_{2}}\left(\hat{\boldsymbol{x}}_{2 p}\right) \otimes Y_{\ell_{1}}\left(\hat{\boldsymbol{x}}_{1 p}\right)\right]_{L L_{z}} N_{[G]}^{(2)} P_{n}^{\ell_{1}, \ell_{2}}\left(\phi_{p}\right) .
$$

Here $Y_{\ell_{1}}\left(\hat{\boldsymbol{x}}_{1 p}\right)$ and $Y_{\ell_{2}}\left(\hat{\boldsymbol{x}}_{2 p}\right)$ are spherical harmonics, $N_{[G]}$ is a normalization factor and ${ }^{(2)} P_{n}^{\ell_{1}, \ell_{2}}\left(\phi_{p}\right)$ is an hyperspherical polynomial. The grand angular quantum number $G$ is defined as $G=2 n+\ell_{1}+\ell_{2}$. The notations $[G]$ and $\{G\}$ of Eqs. (12) and (11) stand for $\left[\ell_{1}, \ell_{2} ; n\right]$ and $\left\{\ell_{1}, \ell_{2}, L, S_{2}, T_{2}\right.$, $S, T ; n\}$, respectively, and $\mu$ of Eq. (9) is $\mu=\{G\}, l$. Note that each set of quantum numbers $\left\{\ell_{1}, \ell_{2}, L, S_{2}, T_{2}, S, T\right\}$ is called "channel", and the antisymmetrization of $\boldsymbol{y}_{\{G\}}\left(\Omega^{(\rho)}\right)$ requires $\ell_{2}+S_{2}+T_{2}$ to be odd. In addition, $\ell_{1}+\ell_{2}$ must be even (odd) for positive (negative) parity.

The $\mathrm{HH}$ functions having grand angular quantum number $G$ constructed in terms of a given set of Jacobi vectors $\boldsymbol{x}_{1 p}, \boldsymbol{x}_{2 p}$, defined starting from the particle order $i, j, k$, can always be expressed in terms of the HH functions constructed, for instance, in terms of $\boldsymbol{x}_{1(p=1)}, \boldsymbol{x}_{2(p=1)}$ with the same value of $G$. In fact, the following relation holds

$$
Y_{\left[\ell_{1}, \ell_{2} ; n\right]}^{L L_{z}}\left(\Omega_{p}^{(\rho)}\right)=\sum_{\ell_{1}^{\prime}, \ell_{2}^{\prime}, n^{\prime}} a_{\ell_{1}, \ell_{2}, n ; \ell_{1}^{\prime}, \ell_{2}^{\prime}, n^{\prime}}^{(p),} Y_{\left[\ell_{1}^{\prime}, \ell_{2}^{\prime} ; n^{\prime}\right]}^{L L_{z}}\left(\Omega_{(p=1)}^{(\rho)}\right),
$$

where the sum is restricted to the values $\ell_{1}^{\prime}, \ell_{2}^{\prime}$, and $n^{\prime}$ such that $\ell_{1}^{\prime}+\ell_{2}^{\prime}+2 n^{\prime}=G$. The coefficients $a_{\ell_{1}, \ell_{2}, n ; \ell_{1}^{\prime}, \ell_{2}^{\prime}, n^{\prime}}^{(p), \text { relat- }}$ ing the two sets of HH functions are known as the RaynalRevai coefficients [25]. Also the spin-isospin states can be recoupled to obtain states where the spin and isospin quantum numbers are coupled in a given order of the particles.
The result is that the antisymmetric functions $\mathcal{Y}_{\{G\}}$ can be expressed as a superposition of functions constructed in terms of a given order of particles $i, j, k$, each one having the pair $i, j$ in a definite spin and angular momentum state. When the two-body potential acts on the pair of particles $i, j$, the effect of the projection is easily taken into account.

The expansion states of Eq. (1) in momentum space can be obtained as follows. Let $\hbar \boldsymbol{k}_{1 p}, \hbar \boldsymbol{k}_{2 p}$ be the conjugate Jacobi momenta of the Jacobi vectors, given by

$$
\begin{aligned}
& \hbar \boldsymbol{k}_{2 p}=\frac{1}{\sqrt{2}}\left(\boldsymbol{p}_{j}-\boldsymbol{p}_{i}\right), \\
& \hbar \boldsymbol{k}_{1 p}=\sqrt{\frac{2}{3}}\left(\boldsymbol{p}_{k}-\frac{1}{2}\left(\boldsymbol{p}_{i}+\boldsymbol{p}_{j}\right)\right),
\end{aligned}
$$

$\boldsymbol{p}_{i}$ being the momentum of the $i$-th particle. We then define a hypermomentum $Q$ and a set of angular-hyperangular variables as

$$
\begin{aligned}
Q & =\sqrt{\boldsymbol{k}_{1 p}^{2}+\boldsymbol{k}_{2 p}^{2}}, \\
\Omega_{p}^{(Q)} & =\left[\hat{\boldsymbol{k}}_{2 p}, \hat{\boldsymbol{k}}_{1 p} ; \varphi_{p}\right],
\end{aligned}
$$

where

$$
\tan \varphi_{p}=\frac{k_{1 p}}{k_{2 p}} .
$$

Then, the momentum-space version of the wave function given in Eq. (9) is

$$
\left|\Psi_{\mu}^{(Q)}\right\rangle=g_{G, l}(Q) \mathcal{Y}_{\{G\}}\left(\Omega^{(Q)}\right),
$$

where $\mathcal{Y}_{\{G\}}\left(\Omega^{(Q)}\right)$ is the same as $\mathcal{Y}_{\{G\}}\left(\Omega^{(\rho)}\right)$ of Eq. (11) with $\boldsymbol{x}_{i p} \rightarrow \boldsymbol{k}_{i p}$, and

$$
g_{G, l}(Q)=(-i)^{G} \int_{0}^{\infty} d \rho \frac{\rho^{3}}{Q^{2}} J_{G+2}(Q \rho) f_{l}(\rho) .
$$

With the adopted form of $f_{l}(\rho)$ given in Eq. (10), the corresponding functions $g_{G, l}(Q)$ can be easily calculated, and they are explicitly given in Ref. [21].

\subsection{The HH Method for Scattering States Below Deuteron Breakup Threshold}

We consider here the extension of the $\mathrm{HH}$ technique to describe $N-d$ scattering states below deuteron breakup threshold, when both local and non-local interaction models are considered.

The wave function $\Psi_{N-d}^{L S J J_{z}}$ describing the $N-d$ scattering state with incoming orbital angular momentum $L$ and channel spin $S$, parity $\pi=(-)^{L}$, and total angular momentum $J, J_{z}$, can be written as

$$
\Psi_{N-d}^{L S J J_{z}}=\Psi_{C}^{L S J J_{z}}+\Psi_{A}^{L S J J_{z}}
$$

where $\Psi_{C}^{L S J J_{z}}$ describes the system in the region where the particles are close to each other and their mutual interactions are strong, while $\Psi_{A}^{L S J J_{z}}$ describes the relative motion between the nucleon $N$ and the deuteron in the asymptotic 
region, where the $N-d$ nuclear interaction is negligible. The function $\Psi_{C}^{L S J J_{z}}$, which has to vanish in the limit of large intercluster separations, can be expanded on the $\mathrm{HH}$ basis as it has been done in the case of bound states. Therefore, applying Eq. (1), the function $\Psi_{C}^{L S J J_{z}}$ can be casted in the form

$$
\left|\Psi_{C}^{L S J J_{z}}\right\rangle=\sum_{\mu} c_{\mu}\left|\Psi_{\mu}\right\rangle
$$

where $\left|\Psi_{\mu}\right\rangle$ is defined in Eqs. (9) and (17) in coordinateand momentum-space, respectively.

The function $\Psi_{A}^{L S J J_{z}}$ is the appropriate asymptotic solution of the relative $N-d$ Schrödinger equation. It is written as a linear combination of the following functions,

$$
\Omega_{L S J J_{z}}^{\lambda}=\sum_{p=1}^{3} \Omega_{L S J J_{z}}^{\lambda}(p),
$$

where the sum over $p$ has to be done over the three even permutations and

$$
\begin{aligned}
\Omega_{L S J J_{z}}^{\lambda}(p)= & \sum_{l=0,2} w_{l}\left(x_{2 p}\right) R_{L}^{\lambda}\left(y_{p}\right)\left\{\left[\left[Y_{l}\left(\hat{\boldsymbol{x}}_{2 p}\right) \otimes S_{2}\right]_{1} \otimes \frac{1}{2}\right]_{S}\right. \\
& \left.\otimes Y_{L}\left(\hat{\boldsymbol{y}}_{p}\right)\right\}_{J J_{z}}\left[T_{2} \otimes \frac{1}{2}\right]_{T T_{z}} .
\end{aligned}
$$

Here the spin and isospin quantum numbers of particles $i$ and $j$ have been coupled to $S_{2}$ and $T_{2}$, with $S_{2}=1$, $T_{2}=0$ for the deuteron, $w_{l}\left(x_{2 p}\right)$ is the deuteron wave function component in the waves $l=0,2, \boldsymbol{y}_{p}$ is the distance between $N$ and the center of mass of the deuteron, i.e. $\boldsymbol{y}_{p}=\sqrt{\frac{3}{2}} \boldsymbol{x}_{1 p}, Y_{l}\left(\hat{\boldsymbol{x}}_{2 p}\right)$ and $Y_{L}\left(\hat{\boldsymbol{y}}_{p}\right)$ are the standard spherical harmonic functions, and the functions $R_{L}^{\lambda}\left(y_{p}\right)$ are the regular $(\lambda \equiv R)$ and irregular $(\lambda \equiv I)$ radial solutions of the relative two-body $N-d$ Schrödinger equation without the nuclear interaction. These regular and irregular functions, denoted as $\mathcal{F}_{L}\left(y_{p}\right)$ and $\mathcal{G}_{L}\left(y_{p}\right)$ respectively, have the form

$$
\begin{aligned}
& \mathcal{F}_{L}\left(y_{p}\right)=\frac{1}{(2 L+1) ! ! q^{L} C_{L}(\eta)} \frac{F_{L}\left(\eta, \xi_{p}\right)}{\xi_{p}} \\
& \mathcal{G}_{L}\left(y_{p}\right)=(2 L+1) ! ! q^{L+1} C_{L}(\eta) f_{R}\left(y_{p}\right) \frac{G_{L}\left(\eta, \xi_{p}\right)}{\xi_{p}}
\end{aligned}
$$

where $q$ is the modulus of the $N-d$ relative momentum (related to the total kinetic energy in the center of mass system by $T_{c m}=\frac{q^{2}}{2 \mu}, \mu$ being the $N-d$ reduced mass), $\eta=2 \mu e^{2} / q$ and $\xi_{p}=q y_{p}$ are the usual Coulomb parameters, and the regular (irregular) Coulomb function $F_{L}\left(\eta, \xi_{p}\right)$ $\left(G_{L}\left(\eta, \xi_{p}\right)\right)$ and the factor $C_{L}(\eta)$ are defined in the standard way [26]. The factor $(2 L+1) ! ! q^{L} C_{L}(\eta)$ has been introduced so that $\mathcal{F}$ and $\mathcal{G}$ have a finite limit for $q \rightarrow 0$. The function $f_{R}\left(y_{p}\right)=\left[1-\exp \left(-b y_{p}\right)\right]^{2 L+1}$ has been introduced to regularize $G_{L}$ at small values of $y_{p}$. The trial parameter $b$ is determined by requiring that $f_{R}\left(y_{p}\right) \rightarrow 1$ outside the range of the nuclear interaction, thus not modifying the asymptotic behaviour of the scattering wave function. A value of $b=0.25 \mathrm{fm}^{-1}$ has been found appropriate. The nonCoulomb case of Eq. (23) is obtained in the limit $e^{2} \rightarrow 0$.
In this case, $F_{L}\left(\eta, \xi_{p}\right) / \xi_{p}$ and $G_{L}\left(\eta, \xi_{p}\right) / \xi_{p}$ reduce to the regular and irregular Riccati-Bessel functions and the factor $(2 L+1) ! ! C_{L}(\eta) \rightarrow 1$ for $\eta \rightarrow 0$. form

With the above definitions, $\Psi_{A}^{L S J J_{z}}$ can be written in the

$$
\Psi_{A}^{L S J J_{z}}=\sum_{L^{\prime} S^{\prime}}\left[\delta_{L L^{\prime}} \delta_{S S^{\prime}} \Omega_{L^{\prime} S^{\prime} J J_{z}}^{R}+\mathcal{R}_{L S, L^{\prime} S^{\prime}}^{J}(q) \Omega_{L^{\prime} S^{\prime} J J_{z}}^{I}\right],
$$

where the parameters $\mathcal{R}_{L S, L^{\prime} S^{\prime}}^{J}(q)$ give the relative weight between the regular and irregular components of the wave function. They are closely related to the reactance matrix $(\mathcal{K}$-matrix) elements, which can be written as

$$
\begin{aligned}
& \mathcal{K}_{L S, L^{\prime} S^{\prime}}^{J}(q)= \\
& (2 L+1) ! !\left(2 L^{\prime}+1\right) ! ! q^{L+L^{\prime}+1} C_{L}(\eta) C_{L^{\prime}}(\eta) \mathcal{R}_{L S, L^{\prime} S^{\prime}}^{J}(q)
\end{aligned}
$$

By definition of the $\mathcal{K}$-matrix, its eigenvalues are $\tan \delta_{L S J}$, $\delta_{L S J}$ being the phase shifts. The sum over $L^{\prime}$ and $S^{\prime}$ in Eq. (24) is over all values compatible with a given $J$ and parity $\pi$. In particular, the sum over $L^{\prime}$ is limited to include either even or odd values since $(-1)^{L^{\prime}}=\pi$.

The matrix elements $\mathcal{R}_{L S, L^{\prime} S^{\prime}}^{J}(q)$ and the linear coefficients $c_{\mu}$ occurring in the expansion of $\Psi_{C}^{L S J J_{z}}$ of Eq. (20) are determined applying the Kohn variational principle, which states that the functional

$$
\begin{aligned}
{\left[\mathcal{R}_{L S, L^{\prime} S^{\prime}}^{J}(q)\right] } & =\mathcal{R}_{L S, L^{\prime} S^{\prime}}^{J}(q)-\left\langle\Psi_{N-d}^{L^{\prime} S^{\prime} J J_{z}}|\mathcal{L}| \Psi_{N-d}^{L S J J_{z}}\right\rangle, \\
\mathcal{L} & =\frac{m}{2 \sqrt{3} \hbar^{2}}(H-E),
\end{aligned}
$$

has to be stationary with respect to variations of the trial parameters in $\Psi_{N-d}^{L S J J_{z}}$. Here $E$ is the total energy of the system, $m$ is the nucleon mass, and $\mathcal{L}$ is chosen so that

$$
\left\langle\Omega_{L S J J_{z}}^{R}|\mathcal{L}| \Omega_{L S J_{z}}^{I}\right\rangle-\left\langle\Omega_{L S J J_{z}}^{I}|\mathcal{L}| \Omega_{L S J J_{z}}^{R}\right\rangle=1 .
$$

As described in Ref. [18], using Eqs. (20) and (24), the variation of the diagonal functionals of Eq. (26) with respect to the linear parameters $c_{\mu}$ leads to the following system of linear inhomogeneous equations:

$$
\sum_{\mu^{\prime}}\left\langle\Psi_{\mu}|\mathcal{L}| \Psi_{\mu^{\prime}}\right\rangle c_{\mu^{\prime}}=-D_{L S J J_{z}}^{\lambda}(\mu) .
$$

Two different terms $D^{\lambda}$ corresponding to $\lambda \equiv R, I$ are introduced and are defined as

$$
D_{L S J J_{z}}^{\lambda}(\mu)=\left\langle\Psi_{\mu}|\mathcal{L}| \Omega_{L S J J_{z}}^{\lambda}\right\rangle .
$$

The matrix elements $\mathcal{R}_{L S, L^{\prime} S^{\prime}}^{J}(q)$ are obtained varying the diagonal functionals of Eq. (26) with respect to them. This leads to the following set of algebraic equations

$$
\sum_{L^{\prime \prime} S^{\prime \prime}} \mathcal{R}_{L S, L^{\prime \prime} S^{\prime \prime}}^{J}(q) X_{L^{\prime} S^{\prime}, L^{\prime \prime} S^{\prime \prime}}=Y_{L S, L^{\prime} S^{\prime}}
$$

with the coefficients $X$ and $Y$ defined as

$$
\begin{aligned}
& X_{L S, L^{\prime} S^{\prime}}=\left\langle\Omega_{L S J J_{z}}^{I}+\Psi_{C}^{L S J J_{z}, I}|\mathcal{L}| \Omega_{L^{\prime} S^{\prime} J J_{z}}^{I}\right\rangle, \\
& Y_{L S, L^{\prime} S^{\prime}}=-\left\langle\Omega_{L S J J_{z}}^{R}+\Psi_{C}^{L S J J_{z}, R}|\mathcal{L}| \Omega_{L^{\prime} S^{\prime} J J_{z}}^{I}\right\rangle .
\end{aligned}
$$




\section{$19^{\text {th }}$ International IUPAP Conference on Few-Body Problems in Physics}

Here $\Psi_{C}^{L S J J_{z}, \lambda}$ is the solution of the set of Eq. (28) with the corresponding $D^{\lambda}$ term. A second order estimate of $\mathcal{R}_{L S, L^{\prime} S^{\prime}}^{J}(q)$ is given by the quantities $\left[\mathcal{R}_{L S, L^{\prime} S^{\prime}}^{J}(q)\right]$, obtained by substituting in Eq. (26) the first order results. Such secondorder calculation provides a symmetric reactance matrix. This condition is not a priori imposed, and therefore it is a useful test of the numerical accuracy.

In the particular case of $q=0$ (zero-energy scattering), the scattering can occur only in the channel $L=0$ and the observables of interest are the scattering lengths. Within the present approach, they can be easily obtained from the relation

$$
{ }^{(2 J+1)} a_{N d}=-\lim _{q \rightarrow 0} \mathcal{R}_{0 J, 0 J}^{J}(q) .
$$

An alternative way to solve the scattering problem, used when $q \neq 0$, is to apply the complex Kohn variational principle to the $\mathcal{S}$-matrix, as in Ref. [18].

The approach presented so far for bound and scattering states does not have too many differences compared to the method presented for instance in Ref. [17], and known as pair-correlated hyperspherical harmonics (PHH) method. In fact, in the $\mathrm{PHH}$ method a correlation factor is included in the $\mathrm{HH}$ expansion of Eq. (20) to take into account the strong short-range correlations induced by the realistic twobody potentials, like the AV18. The presence of correlation functions makes the convergence of the expansion much faster than in the uncorrelated case. However, the PHH method cannot be simply implemented when non-local twobody interactions are considered, unless the Fourier transform of the potential is performed. The calculation involving $\Psi_{C}^{L S J J_{z}}$ can be performed with the $\mathrm{HH}$ or PHH expansions in coordinate- or in momentum-space, depending on what is more convenient.

\section{Three Nucleon Force Models}

In Ref. [13] the description of bound states and zero-energy states for $A=3,4$ has been reviewed in the context of the HH method. In Table 1 we report results for the triton and ${ }^{4} \mathrm{He}$ binding energies as well as for the doublet $n-d$ scattering length ${ }^{2} a_{n d}$ using the AV18 and the N3LO-Idaho NN potentials and using the following combinations of twoand three-nucleon interactions: AV18+URIX, AV18+TM', N3LO-Idaho+N2LOL and N3LO-Idaho+URIXp. In this last model the parameter in front of the spin-isospin independent part of the URIX potential has been rescaled by a factor of 0.384 to fit the triton binding energy [14] (we call this model URIXp). We have considered also the $V_{\text {lowk }}$ model, obtained from the AV18 interaction with a cutoff parameter $\Lambda=2.2 \mathrm{fm}^{-1}$. The results are compared to the experimental values reported in the table. Worthy of notice is the recent very accurate datum for ${ }^{2} a_{n d}$ [27].

From the table we may observe that only the results obtained using an interaction model that includes a TNF are close to the corresponding experimental values. In the case of the AV18+TM', the strength of the TM' potential has been fixed to reproduce the ${ }^{4} \mathrm{He}$ binding energy and, as can be seen from the table, the triton binding energy is underpredicted. Conversely, the strength of the URIX potential
Table 1. The triton and ${ }^{4} \mathrm{He}$ binding energies $B$ (in $\mathrm{MeV}$ ), and doublet scattering length ${ }^{2} a_{n d}$ (in fm) calculated using the indicated two- and three-nucleon interactions. The experimental results are also reported.

\begin{tabular}{llll}
\hline Potential & $B\left({ }^{3} \mathrm{H}\right)$ & $B\left({ }^{4} \mathrm{He}\right)$ & ${ }^{2} a_{n d}$ \\
\hline AV18 & 7.624 & 24.22 & 1.258 \\
N3LO-Idaho & 7.854 & 25.38 & 1.100 \\
AV18+TM' & 8.440 & 28.31 & 0.623 \\
AV18+URIX & 8.479 & 28.48 & 0.578 \\
N3LO-Idaho+N2LOL & 8.474 & 28.37 & 0.675 \\
N3LO-Idaho+URIXp & 8.481 & 28.53 & 0.623 \\
$V_{\text {low- } k}$ & 8.477 & 29.15 & 0.572 \\
\hline Exp. & 8.482 & 28.30 & $0.645 \pm 0.003 \pm 0.007$ \\
\hline
\end{tabular}

has been fixed to reproduce the triton binding energy giving too much binding for ${ }^{4} \mathrm{He}$. The strength of the N2LOL potential has been fixed to reproduce simultaneously the triton and the ${ }^{4} \mathrm{He}$ binding energies whereas the N3LOIdaho+URIXp model overbinds ${ }^{4} \mathrm{He}$. These two models give a better description of ${ }^{2} a_{n d}$. The $V_{l o w-k}$ interaction reproduces the triton binding energy but overbinds ${ }^{4} \mathrm{He}$ appreciably and ${ }^{2} a_{n d}$ is not well described. In conclusion a simultaneous correct description of the three quantities is not achieved by any of the models considered.

To analyze further this fact, we give a brief description of the TM' (or Brazil), URIX and N2LOL models. They can be put in the following way:

$$
\begin{aligned}
W(1,2,3)= & a W_{a}(1,2,3)+b W_{b}(1,2,3)+d W_{d}(1,2,3) \\
W(1,2,3)= & a W_{a}(1,2,3)+b W_{b}(1,2,3)+d W_{d}(1,2,3) \\
& +c_{D} W_{D}(1,2,3)+c_{E} W_{E}(1,2,3) .
\end{aligned}
$$

Each term corresponds to a different source and has a different operator structure. The first three terms arise from the exchange of two pions between three nucleons. The $a$-term is coming from $\pi N S$-wave scattering whereas the $b$-term and $d$-term, which are the most important, come from $\pi N P$-wave scattering. The specific form of these three terms in configuration space is the following:

$$
\begin{aligned}
W_{a}(1,2,3) & =\frac{W_{0}}{c^{2} \hbar^{2}}\left(\boldsymbol{\tau}_{1} \cdot \boldsymbol{\tau}_{2}\right)\left(\boldsymbol{\sigma}_{1} \cdot \boldsymbol{r}_{31}\right)\left(\boldsymbol{\sigma}_{2} \cdot \boldsymbol{r}_{23}\right) y\left(r_{31}\right) y\left(r_{23}\right) \\
W_{b}(1,2,3) & =W_{0}\left(\boldsymbol{\tau}_{1} \cdot \boldsymbol{\tau}_{2}\right)\left[\left(\boldsymbol{\sigma}_{1} \cdot \boldsymbol{\sigma}_{2}\right) y\left(r_{31}\right) y\left(r_{23}\right)\right. \\
& +\left(\boldsymbol{\sigma}_{1} \cdot \boldsymbol{r}_{31}\right)\left(\boldsymbol{\sigma}_{2} \cdot \boldsymbol{r}_{23}\right)\left(\boldsymbol{r}_{31} \cdot \boldsymbol{r}_{23}\right) t\left(r_{31}\right) t\left(r_{23}\right) \\
& +\left(\boldsymbol{\sigma}_{1} \cdot \boldsymbol{r}_{31}\right)\left(\boldsymbol{\sigma}_{2} \cdot \boldsymbol{r}_{31}\right) t\left(r_{31}\right) y\left(r_{23}\right) \\
& \left.+\left(\boldsymbol{\sigma}_{1} \cdot \boldsymbol{r}_{32}\right)\left(\boldsymbol{\sigma}_{2} \cdot \boldsymbol{r}_{32}\right) y\left(r_{31}\right) t\left(r_{23}\right)\right] \\
W_{d}(1,2,3) & =W_{0}\left(\boldsymbol{\tau}_{3} \cdot \boldsymbol{\tau}_{1} \times \boldsymbol{\tau}_{2}\right)\left[\left(\boldsymbol{\sigma}_{3} \cdot \boldsymbol{\sigma}_{2} \times \boldsymbol{\sigma}_{1}\right) y\left(r_{31}\right) y\left(r_{23}\right)\right. \\
& +\left(\boldsymbol{\sigma}_{1} \cdot \boldsymbol{r}_{31}\right)\left(\boldsymbol{\sigma}_{2} \cdot \boldsymbol{r}_{23}\right)\left(\boldsymbol{\sigma}_{3} \cdot \boldsymbol{r}_{31} \times \boldsymbol{r}_{23}\right) t\left(r_{31}\right) t\left(r_{23}\right) \\
& +\left(\boldsymbol{\sigma}_{1} \cdot \boldsymbol{r}_{31}\right)\left(\boldsymbol{\sigma}_{2} \cdot \boldsymbol{r}_{31} \times \boldsymbol{\sigma}_{3}\right) t\left(r_{31}\right) y\left(r_{23}\right) \\
& \left.+\left(\boldsymbol{\sigma}_{2} \cdot \boldsymbol{r}_{32}\right)\left(\boldsymbol{\sigma}_{3} \cdot \boldsymbol{r}_{32} \times \boldsymbol{\sigma}_{1}\right) y\left(r_{31}\right) t\left(r_{23}\right)\right],
\end{aligned}
$$

with $W_{0}$ an overall strength. The $b$ - and $d$-terms are present in the three models whereas the $a$-term is present in the TM' and N2LOL and not in URIX. In the first two models, the radial functions $y(r)$ and $t(r)$ are obtained from the 
following function

$$
f_{0}(r)=\frac{12 \pi}{m_{\pi}^{3}} \frac{1}{2 \pi^{2}} \int_{0}^{\infty} d q q^{2} \frac{j_{0}(q r)}{q^{2}+m_{\pi}^{2}} F_{\Lambda}(q)
$$

where $m_{\pi}$ is the pion mass and

$$
\left\{\begin{array}{l}
y(r)=\frac{1}{r} f_{0}^{\prime}(r) \\
t(r)=\frac{1}{r} y^{\prime}(r)
\end{array}\right.
$$

The cutoff function $F_{\Lambda}$ in the TM' or Brazil models is taken as $\left[\left(\Lambda^{2}-m_{\pi}^{2}\right) /\left(\Lambda^{2}+q^{2}\right)\right]^{2}$. In the N2LOL model it is taken as $\exp \left(-q^{4} / \Lambda^{4}\right)$. The momentum cutoff $\Lambda$ is a parameter of the model fixing the scale of the problem in momentum space. In the N2LOL, it has been taken $\Lambda=500 \mathrm{MeV}$, whereas in the TM' model the quantity $\Lambda / m_{\pi}$ has been varied to describe the triton or ${ }^{4} \mathrm{He}$ binding energy at fixed values of the constants $a, b$ and $d$. In the literature several cases have been explored with typical values around $\Lambda=5 m_{\pi}$.

In the URIX model the radial dependence of the $b$ - and $d$-terms is given in terms of the functions

$$
\left\{\begin{array}{l}
Y(r)=\mathrm{e}^{-x} / x \xi_{Y} \\
T(r)=\left(1+3 / x+3 / x^{2}\right) Y(r) \xi_{T}
\end{array}\right.
$$

with $x=m_{\pi} r$ and the cutoff functions are defined as $\xi_{Y}=$ $\xi_{T}=\left(1-\mathrm{e}^{-c r^{2}}\right)$, with $c=2.1 \mathrm{fm}^{-2}$. This regularization has been used in the AV18 potential as well. Since the parameters in the URIX model has been determined in conjunction with the AV18 potential, the use of the same regularization was a choice of consistency. The relation between the functions $Y(r), T(r)$ and those of the previous models is

$$
\left\{\begin{array}{l}
Y(r)=y(r)+T(r) \\
T(r)=\frac{r^{2}}{3} t(r)
\end{array}\right.
$$

With the definition given in Eq.(35), the asymptotic behaviour of the functions $f_{0}(r), y(r)$ and $t(r)$ is:

$$
\begin{aligned}
& f_{0}(r \rightarrow \infty) \rightarrow \frac{3}{m_{\pi}^{2}} \frac{\mathrm{e}^{-x}}{x} \\
& y(r \rightarrow \infty) \rightarrow-\frac{3 \mathrm{e}^{-x}}{x^{2}}\left(1+\frac{1}{x}\right) \\
& t(r \rightarrow \infty) \rightarrow \frac{3}{r^{2}} \frac{\mathrm{e}^{-x}}{x}\left(1+\frac{3}{x}+\frac{3}{x^{2}}\right) .
\end{aligned}
$$

In fact, with the normalization chosen for $f_{0}$, the functions $Y$ and $T$ defined from $y$ and $t$ in Eq. (38) and those ones defined in the URIX model in Eq. (37) coincide at large separation distances. Conversely, they have a different short range behavior.

The last two terms in Eq. (33) correspond to a $2 \mathrm{~N}$ contact term with a pion emitted or absorbed ( $D$-term) and to a $3 \mathrm{~N}$ contact interaction (E-term). Their local form, in configuration space, derived from Ref. [9], are

$$
W_{D}(1,2,3)=W_{0}^{D}\left(\tau_{1} \cdot \tau_{2}\right) \times
$$

$$
\begin{aligned}
& \left\{\left(\boldsymbol{\sigma}_{1} \cdot \boldsymbol{\sigma}_{2}\right)\left[y\left(r_{31}\right) Z_{0}\left(r_{23}\right)+Z_{0}\left(r_{31}\right) y\left(r_{23}\right)\right]\right. \\
& +\left(\boldsymbol{\sigma}_{1} \cdot \boldsymbol{r}_{31}\right)\left(\boldsymbol{\sigma}_{2} \cdot \boldsymbol{r}_{31}\right) t\left(r_{31}\right) Z_{0}\left(r_{23}\right) \\
& \left.+\left(\boldsymbol{\sigma}_{1} \cdot \boldsymbol{r}_{32}\right)\left(\boldsymbol{\sigma}_{2} \cdot \boldsymbol{r}_{32}\right) Z_{0}\left(r_{31}\right) t\left(r_{23}\right)\right\} \\
W_{E}(1,2,3) & =W_{0}^{E}\left(\boldsymbol{\tau}_{1} \cdot \boldsymbol{\tau}_{2}\right) Z_{0}\left(r_{31}\right) Z_{0}\left(r_{23}\right) .
\end{aligned}
$$

The constant $W_{0}^{D}, W_{0}^{E}$ fix the strength of these terms. In the case of the URIX model the $E$-term is present without the isospin operator structure and it has been included as purely phenomenological, without justifying its form from a particular exchange mechanism. Its radial dependence has been taken as $Z_{0}(r)=T^{2}(r)$. In the N2LOL model, the function $Z_{0}(r)$ is defined as

$$
Z_{0}(r)=\frac{12 \pi}{m_{\pi}^{3}} \frac{1}{2 \pi^{2}} \int_{0}^{\infty} d q q^{2} j_{0}(q r) F_{\Lambda}(q)
$$

with the same cutoff function used in the definition of $f_{0}$ in Eq. (35), $F_{\Lambda}(q)=\exp \left(-q^{4} / \Lambda^{4}\right)$. In the TM' model the $D$ and $E$-terms are absent.

Each model is now identified from the values assigned to the different constants $a, b, d, c_{D}, c_{E}$. Following Refs. [6, 28 ], in the case of the TM' model, the values of the constants have been chosen as $a=-0.87 m_{\pi}^{-1}, b=-2.58 m_{\pi}^{-3}$, and $d=-0.753 m_{\pi}^{-3}$; the strength $W_{0}=\left(g m_{\pi} / 8 \pi m_{N}\right)^{2} m_{\pi}^{4}$ and the cutoff has been fixed to $\Lambda=4.756 m_{\pi}$ in order to describe correctly $B\left({ }^{4} \mathrm{He}\right)$. In Table 1 the calculations have been done using these values with $g^{2}=197.7, m_{\pi}=139.6$ $\mathrm{MeV}, m_{N} / m_{\pi}=6.726\left(m_{N}\right.$ is the nucleon mass $)$ as given in the original derivation of the TM potential. As mentioned before, this model does not include the $D$ - and $E$-terms.

In the URIX model the $b$ - and $d$-terms are present, however with a fix relative value. The strength of these terms is: $b W_{0}=4 A_{2 \pi}^{P W}$ and $d=b / 4$, with $A_{2 \pi}^{P W}=-0.0293$ $\mathrm{MeV}$. The model includes a purely central repulsive term introduced to compensate the attraction of the previous term, which by itself would produce a large overbinding in infinite nuclear matter. It is defined as

$$
W_{E}^{U R I X}(1,2,3)=A_{R} T^{2}\left(r_{31}\right) T^{2}\left(r_{23}\right)
$$

with $A_{R}=0.0048 \mathrm{MeV}$.

In the N2LOL potential the constants of the $a-, b-, d-$, $D$ - and $E$-terms are defined in the following way:

$$
\begin{gathered}
W_{0}=\frac{1}{12 \pi^{2}}\left(\frac{m_{\pi}}{F_{\pi}}\right)^{4} g_{A}^{2} m_{\pi}^{2} \\
W_{D}^{0}=\frac{1}{12 \pi^{2}}\left(\frac{m_{\pi}}{F_{\pi}}\right)^{4}\left(\frac{m_{\pi}}{\Lambda_{x}}\right) \frac{g_{A} m_{\pi}}{8} \\
W_{E}^{0}=\frac{1}{12 \pi^{2}}\left(\frac{m_{\pi}}{F_{\pi}}\right)^{4}\left(\frac{m_{\pi}}{\Lambda_{x}}\right) m_{\pi}
\end{gathered}
$$

with $a=c_{1} m_{\pi}^{2}, b=c_{3} / 2, d=c_{4} / 4$, and $c_{1}=-0.00081$ $\mathrm{MeV}^{-1}, c_{3}=-0.0032 \mathrm{MeV}^{-1}, c_{4}=-0.0054 \mathrm{MeV}^{-1}$ taken from Ref. [16]. The other two constants, $c_{D}=1.0$ and $c_{E}=-0.029$, have been determined in Ref. [9] from a fit to $B\left({ }^{3} \mathrm{H}\right)$ and $B\left({ }^{4} \mathrm{He}\right)$ using the N3LO-Idaho+N2LOL potential model. The numerical values of the constant entering in $W_{0}, W_{D}^{0}$ and $W_{E}^{0}$ are taken as $m_{\pi}=138 \mathrm{MeV}, F_{\pi}=92.4$ $\mathrm{MeV}, g_{A}=1.29$, and the chiral symmetry breaking scale $\Lambda_{x}=700 \mathrm{MeV}$.

In order to analyze the different short range structure of the TNF models, in Fig. 1 we compare the non-dimensional 


\section{$19^{\text {th }}$ International IUPAP Conference on Few-Body Problems in Physics}

functions $Z_{0}(r), y(r)$ and $T(r)$ for the three models under consideration. In the TM' model using the definition of Eq.(41) and using the corresponding cutoff function we can define:

$$
\begin{aligned}
Z_{0}^{T M}(r) & =\frac{12 \pi}{m_{\pi}^{3}} \frac{1}{2 \pi^{2}} \int_{0}^{\infty} d q q^{2} j_{0}(q r)\left(\frac{\Lambda^{2}-m_{\pi}^{2}}{\Lambda^{2}+q^{2}}\right)^{2} \\
& =\frac{3}{2}\left(\frac{m_{\pi}}{\Lambda}\right)\left(\frac{\Lambda^{2}}{m_{\pi}^{2}}-1\right)^{2} \mathrm{e}^{-\Lambda r} .
\end{aligned}
$$

This function is showed in the first panel of Fig. 1 as a dashed line. From the figure we can see that, in the case of the URIX model, the functions $Z_{0}(r)$ and $y(r)$ go to zero as $r \rightarrow 0$. This is not the case for the other two models and is a consequence of the regularization choice of the $Y$ and $T$ functions adopted in the URIX.

\section{Parametrization Study of the Three Nucleon Forces}

In this section we study possible variations to the parametrization of the TNF models in order to describe the $A=3,4$ binding energies and ${ }^{2} a_{n d}$.

\subsection{Tucson-Melbourne Force}

We first study the TM' potential and we would like to see if, using the AV18+TM' interaction, it is possible to reproduce simultaneously the triton binding energy and the doublet $n-d$ scattering length for some values of the parameters. The $a$-term gives a very small contribution to these quantities, therefore, in the following analysis we maintain it fixed at the value $a=-0.87 m_{\pi}^{-1}$. In Fig. 2, left panel, the doublet $n-d$ scattering length is given as a function of the parameter $b$ (in units of its original value $b=-2.58 \mathrm{~m}_{\pi}^{-3}$ ) for different values of the cutoff $\Lambda$ (in units of $m_{\pi}$ ). The box in the figure includes values compatible with the experimental results. The value of the constant $d$ has been fixed to reproduce the triton binding energy. The corresponding values of the parameter $d$ (in units of its original value $d=-0.753 \mathrm{~m}_{\pi}^{-3}$ ) are given in the right panel as a function of $b$. Each point of the curves in both panels corresponds to a set of parameters that, in connection with the AV18 potential, reproduces the triton binding energy. The variations of the parameters given in Fig. 2 do not exhaust all the possibilities. However we can observe that, with the AV18+TM' potential, there is a very small region in the parameter's phase space available for a simultaneous description of the triton binding energy and the doublet scattering length. This small region corresponds to a big value of $b$ and $d$ results to be almost zero. Moreover, the value of the cutoff $\Lambda$ around $3.8 m_{\pi}$ is smaller than the values usually used with the TM' potential $\left(\Lambda \approx 5 m_{\pi}\right)$.

To be noticed that, for negative values of the parameters $a, b$ and $d$, the TM' potential is attractive. It does not include explicitly a repulsive term. Added to a specific NN potential that underpredicts the three-nucleon binding energy, it supplies the extra binding by fixing appropriately its strength. However, as mentioned in the Introduction, the scattering length is sensitive to the balance between the attractive part and the repulsive part of the complete interaction. Therefore, it seems that supplying only an attraction, fixed to reproduce the triton binding energy, in the case of the TM' interaction it is difficult to reproduce correctly this balance.

As discussed before, the TM' potential is a modification of the original TM potential compatible with chiral symmetry. At the same order (next-to-next-to-leading order) in the chiral effective field theory the $D$ - and $E$-terms appear (see Ref. [8] and references therein) as given in Eq.(33). Here we introduce the following additional term to the TM' potential based on a contact term of three nucleons

$$
W_{E}^{T M}(1,2,3)=W_{E}^{0} \sum_{c y c} Z_{0}^{T M}\left(r_{31}\right) Z_{0}^{T M}\left(r_{23}\right) .
$$

This term is similar to the repulsive term of the URIX model and, for the sake of simplicity, we do not include the $\left(\tau_{1} \cdot \tau_{2}\right)$ operator. The function $Z_{0}^{T M}$ is a positive function, therefore, for positive values of $c_{E}$, the new term is repulsive. We include it in the following analysis of the TM' potential. The analysis of the new term is given in Fig. 3. In the left panel the doublet $n-d$ scattering length is given as a function of the parameter $b$ (in units of its original value $b=-2.58 m_{\pi}^{-3}$ ) for different values of the strength of the $W_{E}^{T M}$-term. The value of the cutoff $\Lambda$ has been fixed to $4.8 m_{\pi}$. The box in the figure includes values compatible with the experimental results. Moreover, the value of the constant $d$ has been fixed to reproduce the triton binding energy. The corresponding values of the ${ }^{4} \mathrm{He}$ binding energy, $B\left({ }^{4} \mathrm{He}\right)$, is given in the right panel.

Comparing the left panels in Figs. 2 and 3, the effect of the new term is clear. In Fig. 2 we see that using $\Lambda=$ $4.8 m_{\pi},{ }^{2} a_{n d}$ is not well reproduced. Conversely, in Fig. 3, the inclusion of the new term moves this curve in the correct direction and with values of its strength around $c_{E}=$ 1.6 it is possible to reproduce the experimental value of ${ }^{2} a_{n d}$. There is also an improvement in the description of $B\left({ }^{4} \mathrm{He}\right)$. In fact, the AV18+TM' model with $\Lambda=4.8 m_{\pi}$ reproduces the triton binding energy as can be seen from Fig. 2. However it predicts $B\left({ }^{4} \mathrm{He}\right)=28.55 \mathrm{MeV}$, which is slightly too high. With the $W_{E}^{T M}$-term, at $c_{E}=1.6$, the description of $B\left({ }^{4} \mathrm{He}\right)$ improves. For example with $b=$ $-3.87 m_{\pi}^{-3}, d=-3.375 m_{\pi}^{-3}$ and $\Lambda=4.8 m_{\pi}$, we obtain $B\left({ }^{4} \mathrm{He}\right)=28.36 \mathrm{MeV}$, very close to the experimental value.

\subsection{UrbanalX Force}

In the following we analyze the URIX potential which has two parameters, $A_{2 \pi}^{P W}$ and $A_{R}$. In this model the strength of the $d$-term was related to the strength of the $b$-term as $b=4 d$. The original values of the parameters were fixed in Ref. [4] in conjunction with the AV18 NN potential 

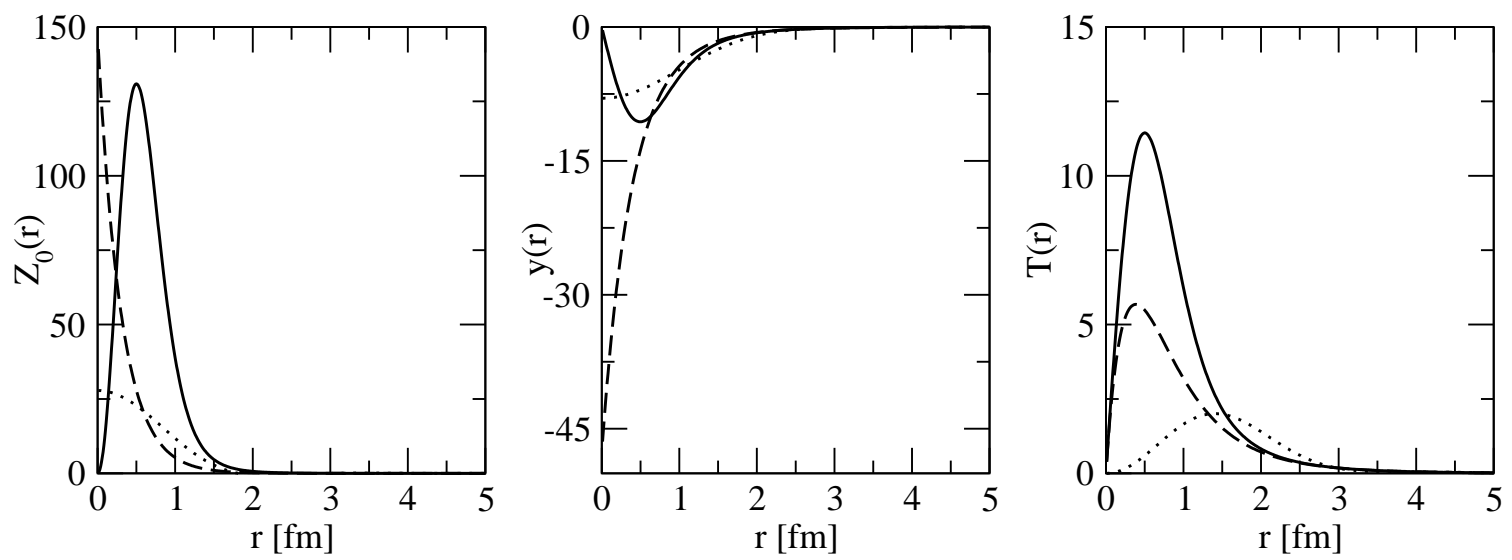

Fig. 1. The $Z_{0}(r), y(r)$ and $T(r)$ functions as functions of the interparticle distance $r$ for the URIX (solid line), TM' (dashed line) and N2LOL (dotted line) models.
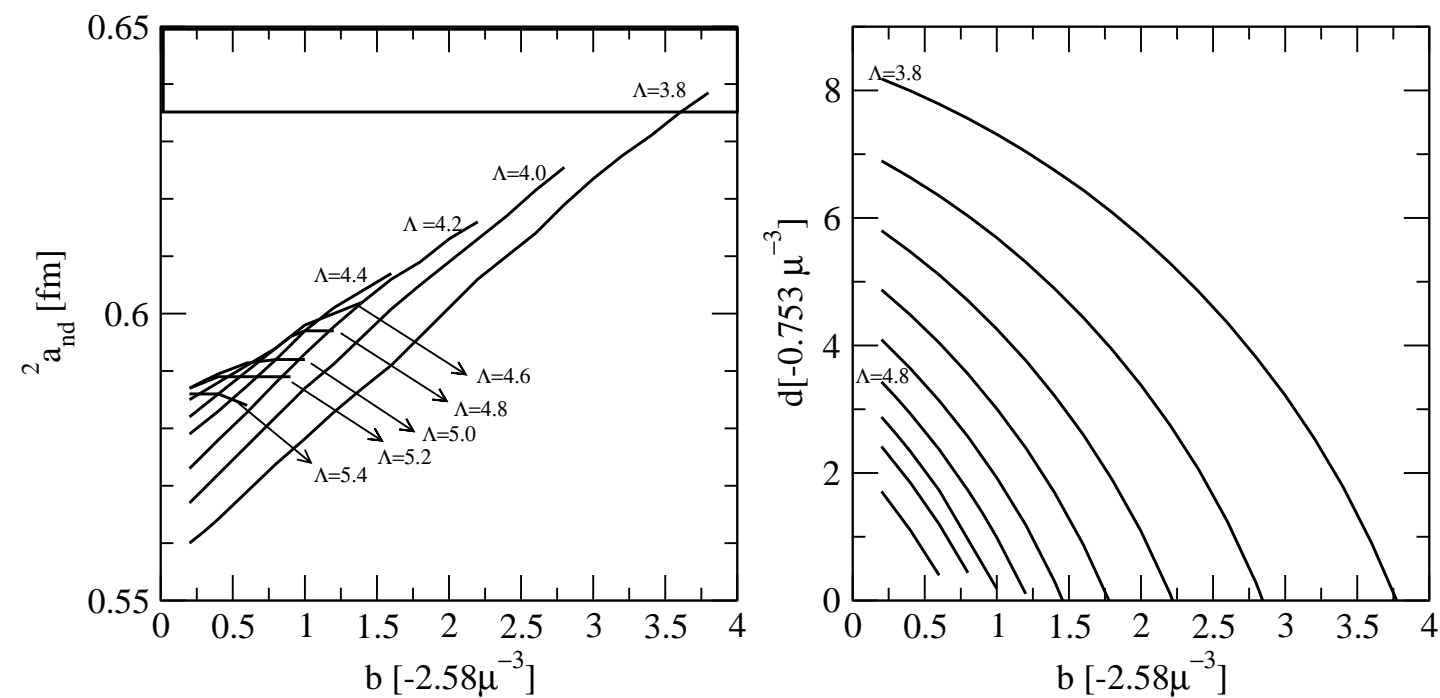

Fig. 2. The doublet scattering length $a_{n-d}$ as a function of the parameter $b$ of the TM' potential (right panel) for different values of the cutoff. The corresponding values of the parameter $d$ used to reproduce the triton binding energy (left panel).

and, from Table 1, we observe that the model correctly describes the triton binding energy. However, it overestimates $B\left({ }^{4} \mathrm{He}\right)$ and underestimates ${ }^{2} a_{n d}$. In order to improve the description of these quantities, we have varied the constants $A_{2 \pi}^{P W}, A_{R}$ and the relative strength $D_{2 \pi}^{P W}=d / b$ between the $b$ - and $d$-terms. For a given value of $A_{2 \pi}^{P W}$, the values of $A_{R}$ and $D_{2 \pi}^{P W}$ has been chosen to reproduce $B\left({ }^{3} \mathrm{H}\right)$ and ${ }^{2} a_{n d}$. The results are given in Fig. 4. In panel (a), $A_{2 \pi}^{P W}$ is given as a function of $D_{2 \pi}^{P W}$ with $A_{R}$ varying from 0.0176
$\mathrm{MeV}$ at $A_{2 \pi}^{P W}=-0.02$ to $0.0210 \mathrm{MeV}$ at $A_{2 \pi}^{P W}=-0.050$ $\mathrm{MeV}$. These values of $A_{R}$ are more than three times greater than the original value. In panel (b) and (c) the results for ${ }^{2} a_{n d}$ and $B\left({ }^{4} \mathrm{He}\right)$ are given respectively. The latter has not been included in the determination of the parameters, however we observe a rather good description in particular for values of $D_{2 \pi}^{P W}>0.7$.

With a modification of the parameters in the URIX force, we were able to describe $B\left({ }^{3} \mathrm{H}\right),{ }^{2} a_{n d}$ and $B\left({ }^{4} \mathrm{He}\right)$. This has been achieved with a substantial increase of the 

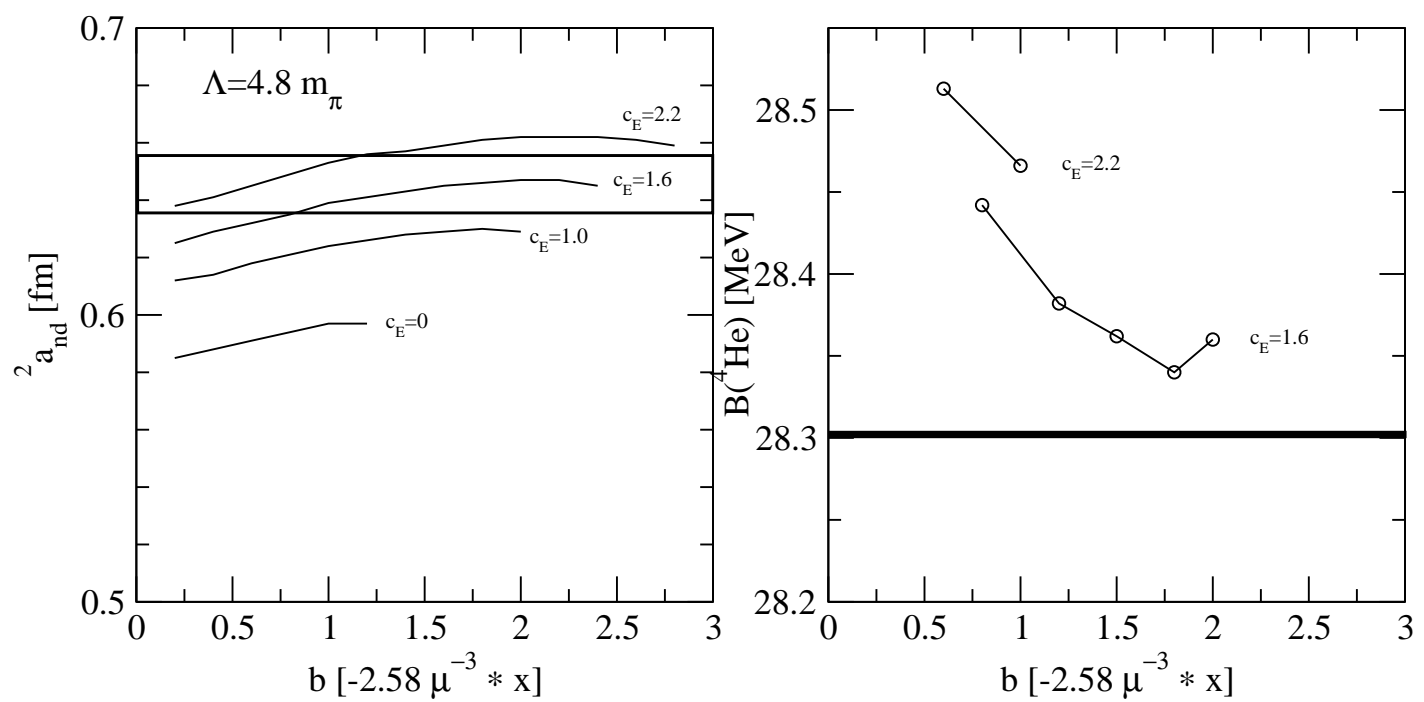

Fig. 3. The doublet scattering length $a_{n-d}$ as a function of the parameter $b$ of the TM' potential including the $W_{E}^{T M}$-term, for different values of the strength $c_{E}$ (right panel). The corresponding values of $B\left({ }^{4} \mathrm{He}\right)$ (left panel).

repulsive term. Also $D_{2 \pi}^{P W}$ is quite far from its original value. For example, at the original value of $A_{2 \pi}^{P W}=-0.0293 \mathrm{MeV}$, the relative strength is $D_{2 \pi}^{P W}=1$ and $A_{R}=0.0181 \mathrm{MeV}$. This is four times and more than three of the original values, respectively. As $D_{2 \pi}^{P W}$ diminishes, $A_{R}$ tends to increase further with the consequence that the mean value of the repulsive part of $W$ results to be more than three times the original AV18+URIX value. This is compensated by a lower mean value of the kinetic energy. A further analysis of the effects of the new parametrizations is done in the next section studying selected $p-d$ polarization observables.

\subsection{N2LOL Force}

The parameters $c_{1}, c_{3}$ and $c_{4}$ of the N2LOL have been taken from the chiral N3LO NN force of Ref. [16], whereas the $c_{D}$ and $c_{E}$ parameters have been determined in Ref. [9], in conjunction with that NN force, by fitting $B\left({ }^{3} \mathrm{H}\right)$ and $B\left({ }^{4} \mathrm{He}\right)$. Here we are going to use the N2LOL force in conjunction with the AV18 NN interaction, so we have to modify its parametrization since the amount of attraction to be gained is now different (see Table 1). Moreover, the modification has to be done in such a way that $B\left({ }^{3} \mathrm{H}\right)$ and ${ }^{2} a_{n d}$ are well reproduced. As an example, in Fig. $5,{ }^{2} a_{n d}$ is shown as a function of the parameter $c_{3}$ (in units of its original value $c_{3}=0.0032 \mathrm{MeV}^{-1}$ ) fixing $c_{D}=0.4, c_{E}=0.1$ and varying $c_{4}$ in order to reproduce $B\left({ }^{3} \mathrm{H}\right)$. With the values $c_{3}=-0.0048 \mathrm{MeV}^{-1}, c_{4}=0.0043 \mathrm{MeV}^{-1},{ }^{2} a_{n d}$ fall inside the box and matches the experimental value. In this case, the ${ }^{4} \mathrm{H}$ binding energy results $B\left({ }^{4} \mathrm{H}\right)=28.36 \mathrm{MeV}$.

\section{Polarization observables with the new parametrizations}

In the previous section we have analyzed different parametrizations of the TM', URIX and N2LOL TNFs determined in conjunction with the AV18 NN potential. With the new parametrizations the three quantities under observation, $B\left({ }^{3} \mathrm{H}\right),{ }^{2} a_{n d}$ and $B\left({ }^{4} \mathrm{He}\right)$, are well reproduced. However, some substantial modifications to the first two models were necessary. In the case of the TM' interaction, we found necessary to include a repulsive term. In the analysis of the URIX interaction, the strength of the repulsive term resulted to be more than three times larger. In the case of the N2LOL interaction, a minor adjustment of the parameters was necessary. Now we would like to analyze the effects of the new parametrizations in observables that are not correlated to the binding energies or to ${ }^{2} a_{n d}$. Some polarization observables in $p-d$ scattering have this characteristic, in particular the vector and tensor analyzing powers. In Fig. 5, the differential cross section $d \sigma / d \Omega$, the vector polarization observables $A_{y}$ and $i T_{11}$ and the tensor polarization observables $T_{20}, T_{21}$ and $T_{22}$ are shown at the laboratory energy $E_{\text {lab }}=3 \mathrm{MeV}$, for the different potential models. As a reference we use the AV18+URIX interaction given in the figure as a blue line. In the figure, the other three curves corresponds to particular parametrizations of the models that reproduce ${ }^{2} a_{n d}$ and $B\left({ }^{3} \mathrm{H}\right)$ and approximate, as much as possible, $B\left({ }^{4} \mathrm{He}\right)$. The parametrizations of the models selected for the figure are the following: the AV18+URIX ${ }^{*}$ model is defined with $A_{2 \pi}^{P W}=-0.0293$ $\mathrm{MeV}, D_{2 \pi}^{P W}=1$ and $A_{R}=0.018 \mathrm{MeV}$. In the AV18+TM* model we have used $a=-0.87 m_{\pi}^{-1}, b=-9.804 m_{\pi}^{-3}$, 

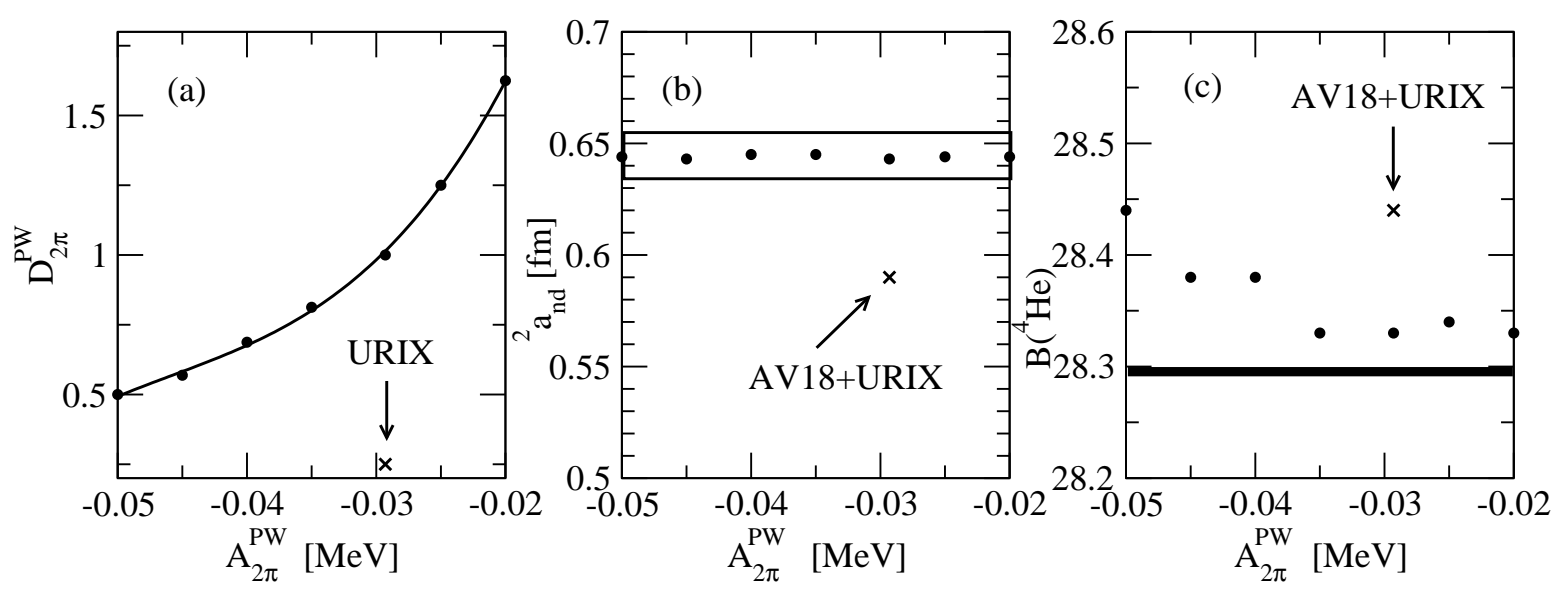

Fig. 4. (a) The relative strength $D_{2 \pi}^{P W}$ as a function of $A_{2 \pi}^{P W}$. In each point of the curve the triton binding energy and ${ }^{2} a_{n d}$ are well described. (b) Values of ${ }^{2} a_{n d}$ for the seven combinations of the parameters indicated as solid points in panel (a). (c) The corresponding predictions for $B\left({ }^{4} \mathrm{He}\right)$. The crosses indicate the results using the parameters defined in the URIX model

$d=-3.1657 m_{\pi}^{-3}, c_{E}=1$, and $\Lambda=4 m_{\pi}$. In the AV18+N2LO* model the parametrization corresponds to $c_{1}=-0.00081 \mathrm{MeV}^{-1}$ (its original value), $c_{3}=-0.0048$ $\mathrm{MeV}^{-1}, c_{4}=-0.0043 \mathrm{MeV}^{-1}, c_{D}=0.4$ and $c_{E}=0.1$. From the figure we can observe that the models describe equally well the differential cross section and the tensor analyzing powers $T_{20}, T_{22}$. Differences are observed in the vector analyzing powers $A_{y}$ and $i T_{11}$. Taking as a reference the results of the AV18+URIX model, in both cases the AV18+URIX* model produces a noticeable worse description whereas the AV18+N2LOL* slightly improves the description. The new parametrizations of the TNF models overpredict $T_{21}$ in all cases, in particular the AV18+TM* model.

\section{The Kohn Variational Principle in terms of Integral Relations}

Recently two integral relations have been derived from the KVP [22]. It has been shown that starting from the KVP, the tangent of the phase-shift can be expressed in a form of a quotient where both, the numerator and the denominator, are given as two integral relations. Let us first consider a two-body system interacting through a short-range potential $V(r)$ at the center of mass energy $E$ in a relative angular momentum state $l=0$. The solution of the Schrödinger equation in configuration space ( $m$ is twice the reduced mass)

$$
\left(-\frac{\hbar^{2}}{m} \nabla^{2}+V-E\right) \Psi(\boldsymbol{r})=0
$$

can be obtained after specifying the corresponding boundary conditions. For $E>0$, with $k^{2}=E /\left(\hbar^{2} / m\right)$ and assum-

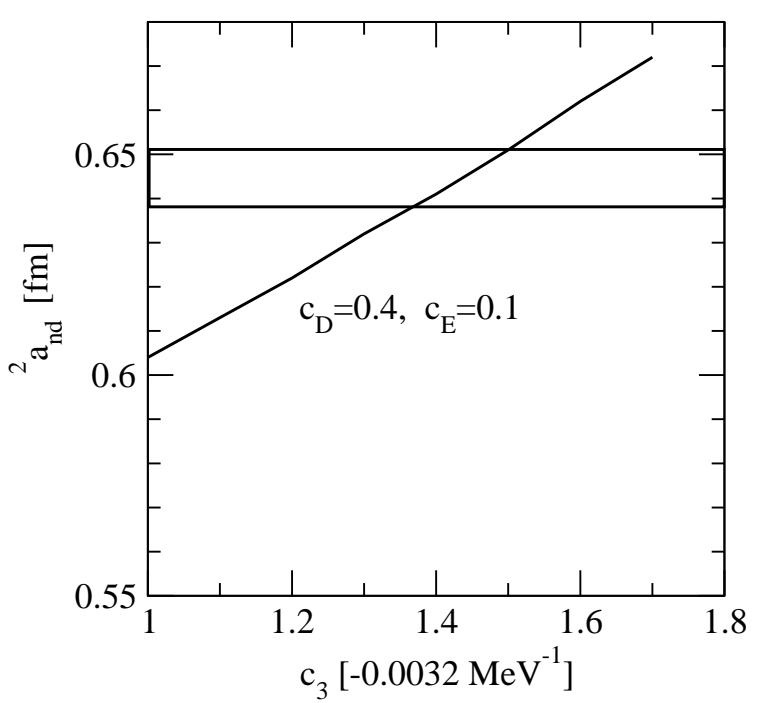

Fig. 5. ${ }^{2} a_{n d}$ as a function of the $c_{3}$ parameter in the N2LOL model.

ing a short-range potential $V, \Psi(\boldsymbol{r})=\phi(r) / \sqrt{4 \pi}$ and

$$
\phi(r \rightarrow \infty) \longrightarrow \sqrt{k}\left[A \frac{\sin (k r)}{k r}+B \frac{\cos (k r)}{k r}\right]
$$

With the above normalization, the solution $\Psi$ verifies the following integral relations:

$$
-\frac{m}{\hbar^{2}}<\Psi|H-E| F>=B \text { with } \quad F=\sqrt{\frac{k}{4 \pi}} \frac{\sin (k r)}{k r}
$$



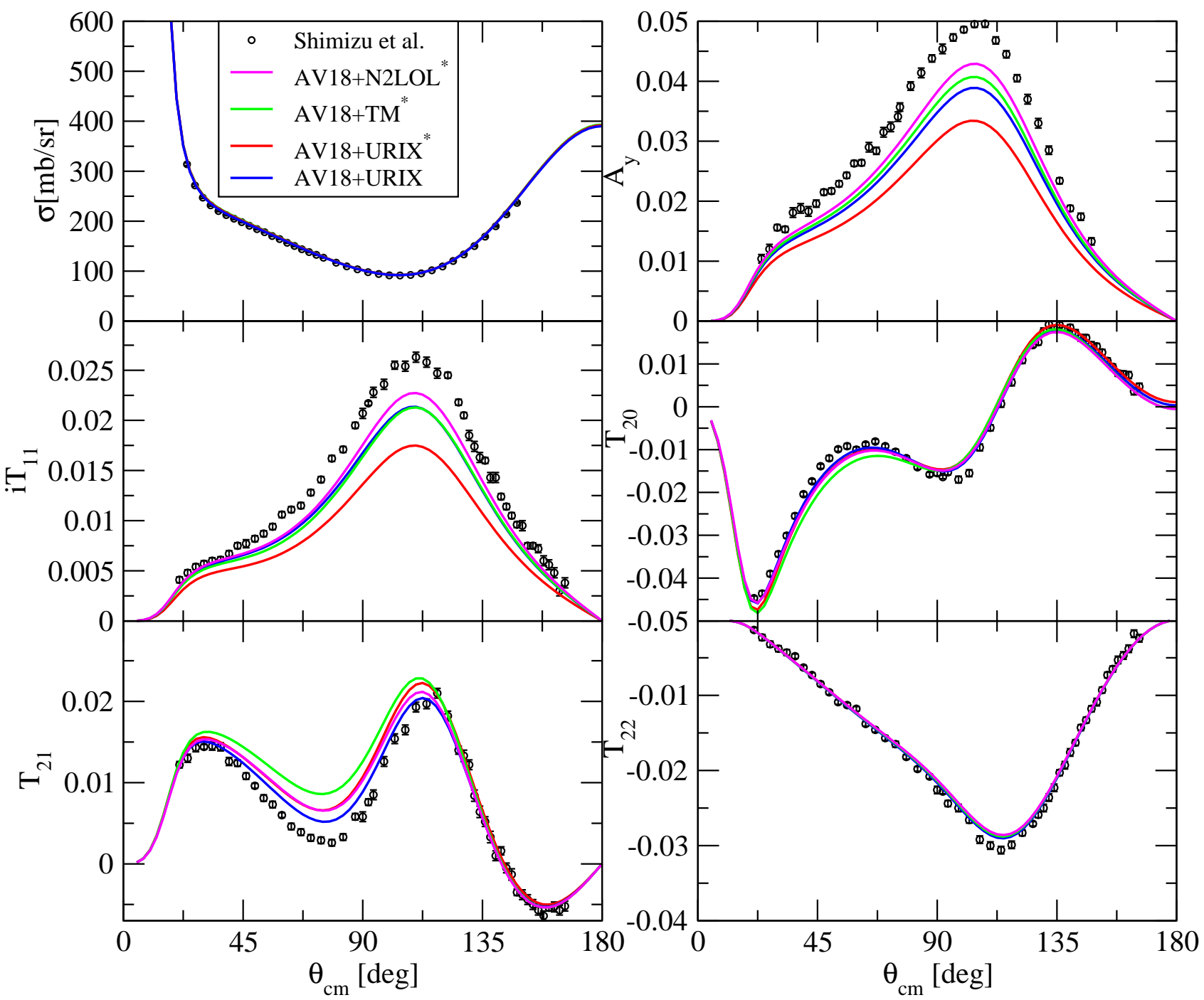

Fig. 6. Cross section, vector and tensor analyzing powers for $p-d$ scattering at $E_{l a b}=3 \mathrm{MeV}$. Experimental points are for Ref. [29]

$$
\begin{aligned}
\frac{m}{\hbar^{2}}< & \Psi|H-E| G>=A \text { with } \quad G=\sqrt{\frac{k}{4 \pi}} \frac{\cos (k r)}{k r} \\
& \tan \delta=\frac{B}{A} .
\end{aligned}
$$

Explicitly they are

$$
\begin{aligned}
- & \frac{m}{\hbar^{2} \sqrt{k}} \int_{0}^{\infty} d r \sin (k r) V(r)[r \phi(r)]=B \\
& \frac{m}{\hbar^{2} \sqrt{k}} \int_{0}^{\infty} d r \cos (k r) V(r)[r \phi(r)]+\frac{\phi(0)}{\sqrt{k}}=A,
\end{aligned}
$$

where in the last integral we have used the property $\nabla^{2}(1 / r)=$ $-4 \pi \delta(r)$.

In practical cases the solution of the Schrödinger equation is obtained numerically. Then, $\tan \delta$ is extracted from $\phi(r)$ analyzing its behavior outside the range of the potential. The equivalence between the extracted value and that one obtained from the integral relations defines the accuracy of the numerical computation. A relative difference of the order of $10^{-7}$ of the two values is usually achieved using standard numerical techniques to solve the differential equation and to compute the two one-dimensional integrals. To be noticed the short range character of the integral relations. This means that the phase-shift is determined by the internal structure of the wave function.

The last relation in Eq. (49) shows a dependence on the value of the wave function at the origin. It could be convenient to eliminate this explicit dependence since the numerical determination of $\phi(0)$ might be problematic, as we will show. To this end we introduce a regularized func- 
tion $\tilde{G}=f_{\text {reg }} G$ with the property $|\tilde{G}(r=0)|<\infty$ and $\tilde{G}=G$ outside the interaction region. A possible choice is

$$
\tilde{G}=\sqrt{\frac{k}{4 \pi}} \frac{\cos (k r)}{k r}\left(1-\mathrm{e}^{-\gamma r}\right)
$$

where the regularization function $f_{\text {reg }}=\left(1-\mathrm{e}^{-\gamma r}\right)$ has been introduced with $\gamma$ being a non linear parameter which will be discussed below. Values verifying $\gamma>1 / r_{0}$, with $r_{0}$ the range of the potential, could be appropriate. The regularized function $\tilde{G}$ (as well as the irregular function $G$ ), verifies the normalization condition

$$
\frac{m}{\hbar^{2}}[<F|H-E| \tilde{G}>-<\tilde{G}|H-E| F>]=1 .
$$

Therefore the second integral relation in Eq. (48) remains valid using $\tilde{G}$ in place of $G$,

$$
\frac{m}{\hbar^{2}}<\Psi|H-E| \tilde{G}>=A,
$$

with the explicit form:

$$
\frac{m}{\hbar^{2} \sqrt{k}} \int_{0}^{\infty} d r \cos (k r) V(r)[r \phi(r)]+I_{\gamma}=A
$$

where in $I_{\gamma}$ all terms depending on $\gamma$, introduced by $f_{\text {reg }}$, are included. Comparing Eq. (53) to Eq. (49) we identify $I_{\gamma}=\phi(0) / \sqrt{k}$.

In the following we demonstrate that the relation $\tan \delta=$ $B / A$, which is an exact relation when the exact wave function $\Psi$ is used in Eq. (48), can be considered accurate up to second order when a trial wave function is used, as it has a strict connection with the Kohn variational principle.

The connection of the integral relations with the KVP is straightforward. Defining a trial wave function $\Psi_{t}$ as

$$
\Psi_{t}=\Psi_{c}+A F+B \tilde{G}
$$

with $\Psi_{c} \rightarrow 0$ as $r \rightarrow \infty$, the condition $\Psi_{t} \rightarrow A F+B G$ as $r \rightarrow \infty$ is fulfilled. The KVP states that the second order estimate for $\tan \delta$ is

$$
[\tan \delta]^{2^{n d}}=\tan \delta-\frac{m}{\hbar^{2}}<(1 / A) \Psi_{t}|H-E|(1 / A) \Psi_{t}>.
$$

The above functional is stationary with respect to variations on $\Psi_{c}$ and $\tan \delta$. Without loosing generality $\Psi_{c}$ can be expanded in a (square integrable) complete basis

$$
\Psi_{c}=\sum_{n} a_{n} \phi_{n}(r)
$$

The variation of the functional with respect to the linear parameters $a_{n}$ and $\tan \delta$ leads to the following equations

$$
\begin{aligned}
& <\phi_{n}|H-E| \Psi_{t}>=0 \\
& <\tilde{G}|H-E| \Psi_{t}>=0 .
\end{aligned}
$$

To obtain the last equation, the normalization relation of Eq. (51) has been used. From these two equations, $\Psi_{c}$ and the first order estimate of the phase shift $(\tan \delta)^{1^{s t}}$ can be determined. To be noticed that the first equation implies $\left.<\Psi_{c}|H-E| \Psi_{t}\right\rangle=0$. Furthermore, from the general relation $\left(m / \hbar^{2}\right)\left[<\Psi_{t}|H-E| \tilde{G}>-<\tilde{G}|H-E| \Psi_{t}>\right]=A$, and using the second equation in Eq. (57), the following integral relation results

$$
\frac{m}{\hbar^{2}}<\Psi_{t}|H-E| \tilde{G}>=A
$$

Replacing the two relations of Eq.(57) into the functional of Eq.(55), a second order estimate of the phase shift is obtained

$$
[\tan \delta]^{2^{\text {nd }}}=(\tan \delta)^{1^{s t}}-\frac{m}{\hbar^{2}}<F|H-E|(1 / A) \Psi_{t}>.
$$

Multiplying Eq. (59) by $A$ one gets

$$
B^{2^{n d}}=B^{1^{s t}}-\frac{m}{\hbar^{2}}<F|H-E| \Psi_{t}>.
$$

On the other hand, a first order estimate for the coefficient $B$ can be obtained from the general relation

$$
\frac{m}{\hbar^{2}}\left[<F|H-E| \Psi_{t}>-<\Psi_{t}|H-E| F>\right]=B^{1^{s t}} .
$$

Therefore, replacing Eq.(61) in Eq.(60), a second order integral relation for $B$ is obtained. The above results can be summarized as follow

$$
\begin{aligned}
B^{2^{n d}} & =-\frac{m}{\hbar^{2}}<\Psi_{t}|H-E| F> \\
A & =\frac{m}{\hbar^{2}}<\Psi_{t}|H-E| \tilde{G}> \\
{[\tan \delta]^{\text {ndd }} } & =B^{2^{n d}} / A .
\end{aligned}
$$

These equations extend the validity of the integral relations, given in Eq.(48) for the exact wave functions, to trial wave functions. To be noticed that $F, \tilde{G}$ are solutions of the Schrödinger equation in the asymptotic region, therefore $(H-E) F \rightarrow 0$ and $(H-E) \tilde{G} \rightarrow 0$ as the distance between the particles increases. As a consequence the decomposition of $\Psi_{t}$ in the three terms of Eq. (54) can be considered formal since, due to the short-range character of the relation integrals, it is sufficient that the trial wave function be a solution of $(H-E) \Psi_{t}=0$ in the interaction region, without an explicit indication of its asymptotic behavior. This fact, together with the variational character of the relations allows for a number of applications to be discussed in the next sections.

\section{Integral Relations for $A=2,3$ systems}

Applications of the integral relations to systems with $A=$ 2,3 are given. We first consider the following central, $s$ wave gaussian potential

$$
V(r)=-V_{0} \exp \left(-r^{2} / r_{0}^{2}\right),
$$

with $V_{0}=-51.5 \mathrm{MeV}, r_{0}=1.6 \mathrm{fm}$ and $\hbar^{2} / m=41.4696$ $\mathrm{MeV} \mathrm{fm}{ }^{2}$. This potential has a shallow $L=0$ bound state with energy $E_{2 B}=-0.397743 \mathrm{MeV}$. 
In the $A=2$ system, the orthogonal basis

$$
\phi_{m}=\mathcal{L}_{m}^{(2)}(z) \exp -(z / 2)
$$

with $\mathcal{L}_{m}$ a (normalized) Laguerre polynomial and $z=\beta r$, being $\beta$ a nonlinear parameter, is used to expand the wave function of the system

$$
\Psi_{0}=\sum_{m=0}^{M-1} a_{m}^{0} \phi_{m}
$$

The Schrödinger equation is transformed to an eigenvalue problem that can be solved for different values of the dimension $M$ of the basis. The variational principle states that

$$
E_{0}=\left\langle\Psi_{0}|H| \Psi_{0}\right\rangle \geq E_{2 B},
$$

with the equality obtained for $M \rightarrow \infty$. The nonlinear parameter $\beta$ can be fixed to make improve the convergence properties of the basis. In fact, for each value of $M$ there is a value of $\beta$ that minimizes the energy. Increasing $M$, the minimum of the energy becomes less dependent on $\beta$ resulting in a plateau. Increasing further the dimension of the basis, the extension of the plateau increases as well, without any appreciable improvement in the eigenvalue, indicating that the convergence has been reached up to certain accuracy. At each step $\Psi_{0}$ represents a first order estimate of the bound state exact wave function.

In the proposed example the system has only one bound state. So, with proper values of $M$ and $\beta$, the diagonalization of $H$ results in one negative eigenvalue $E_{0}$ and $M-1$ positive eigenvalues $E_{j}(j=1, \ldots ., M-1)$. The corresponding wave functions

$$
\Psi_{j}=\sum_{m=0}^{M-1} a_{m}^{j} \phi_{m} \quad j=1, \ldots, M-1,
$$

are approximate solutions of $\left(H-E_{j}\right) \Psi_{j}=0$ in the interaction region. As $r \rightarrow \infty$ they go to zero exponentially and therefore they do not represent a physical scattering state. The negative energy $E_{0}$ and the first three positive energy eigenvalues $\left(E_{j}, j=1,3\right)$ are shown in Fig. 7 as a function of $\beta$ in the case of $M=40$. We observe the plateau already reached by $E_{0}$ for the values of $\beta$ showed in the figure. We observe also the monotonic behavior of the positive eigenvalues toward zero as $\beta$ decreases. The corresponding eigenvectors can be used to compute the integral relations of Eq. (62) and to calculate the second order estimate of the phase-shifts $\delta_{j}$ at the specific energies $E_{j}$. This analysis is shown in Table 2 in which the non linear parameter $\beta$ of the Laguerre basis has been fixed to $1.2 \mathrm{fm}^{-1}$. In the first row of the table the ground state energy is given for different values of the number $M$ of Laguerre polynomials. The stability of $E_{0}$ at the level of $1 \mathrm{keV}$ is achieved already with $M=20$. For a given value of $M, E_{j}$, with $j=1,2,3$, are the first three positive eigenvalues. The eigenvectors corresponding to positive energies approximate the scattering states at the specific energies. Since the lowest scattering state appears at zero energy, none of the positive eigenvalues can reach this value for any finite values of $M$. Defining

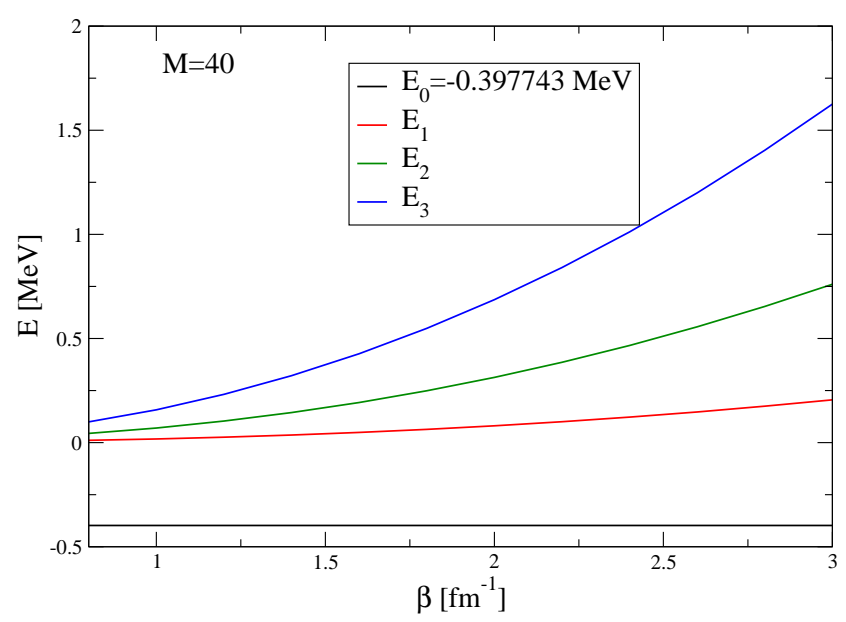

Fig. 7. The two-nucleon bound state energy $E_{0}$ and the first three positive eigenvalues $E_{j}$ as a function of $\beta$ in the case of $M=40$

$k_{j}^{2}=\frac{m}{\hbar^{2}} E_{j}$, the second order estimate for the phase shift at each energy and at each value of $M$ is obtained as

$$
\begin{gathered}
-\frac{m}{\hbar^{2}}<\Psi_{j}|H-E| F_{j}>=B_{j} \text { with } F_{j}=\sqrt{\frac{k_{j}}{4 \pi}} \frac{\sin \left(k_{j} r\right)}{k_{j} r} \\
\frac{m}{\hbar^{2}}<\Psi_{j}|H-E| \tilde{G}_{j}>=A_{j} \text { with } \tilde{G}_{j}=f_{r e g} \sqrt{\frac{k_{j}}{4 \pi}} \frac{\cos \left(k_{j} r\right)}{k_{j} r} \\
{\left[\tan \delta_{j}\right]^{2^{n d}}=B_{j} / A_{j} .}
\end{gathered}
$$

Table 2. The two-nucleon bound state $E_{0}$ and the first three positive eigenvalues $E_{j}(j=1,3)$, as a function of the number of Laguerre polynomials $M$. The second order estimates, $\left[\tan \delta_{j}\right]^{2^{n d}}$, obtained applying the integral relations are given in each case and compared to exact results, $\tan \delta_{j}$.

\begin{tabular}{lrrrr}
\hline $\mathrm{M}$ & 10 & 20 & 30 & 40 \\
\hline$E_{0}$ & -0.395079 & -0.397740 & -0.397743 & -0.397743 \\
\hline$E_{1}$ & 0.536349 & 0.116356 & 0.048091 & 0.026008 \\
{$\left[\tan \delta_{1}\right]^{2^{n d}}$} & -1.507280 & -0.622242 & -0.392005 & -0.286479 \\
$\tan \delta_{1}$ & -1.522377 & -0.621938 & -0.392021 & -0.286480 \\
\hline$E_{2}$ & 1.984580 & 0.449655 & 0.190019 & 0.103503 \\
{$\left[\tan \delta_{2}\right]^{2^{n d}}$} & -5.919685 & -1.353736 & -0.812313 & -0.584389 \\
$\tan \delta_{2}$ & -5.703495 & -1.354691 & -0.812270 & -0.584388 \\
\hline$E_{3}$ & 4.512635 & 0.994433 & 0.423117 & 0.231645 \\
{$\left[\tan \delta_{3}\right]^{2^{n d}}$} & 13.998124 & -2.451174 & -1.302799 & -0.908128 \\
$\tan \delta_{3}$ & 12.684474 & -2.448343 & -1.302887 & -0.908131 \\
\hline
\end{tabular}

On the other hand, as we are considering the $A=2$ system, at each specified energy $E_{j}$ the phase shift $\tan \delta_{j}$ can be obtained by solving the Schrödinger equation numerically. The two values, $\left[\tan \delta_{j}\right]^{2^{n d}}$ and $\tan \delta_{j}$, are given 
in the Table 2 at the corresponding energies as a function of $M$. We observe that, as $M$ increases, the relative difference between the variational estimate and the exact value reduces, for example at $M=40$ is around $10^{-6}$. In fact, as $M$ increases, each eigenvector gives a better representation of the exact wave function in the internal region and the second order estimates, $\left[\tan \delta_{j}\right]^{2^{\text {nd }}}$ approach the exact result.

In a different application, the integral relations can be used to calculate the phase-shift of a process in which the two particles interact through a short range potential plus the Coulomb potential, imposing free asymptotic conditions to the wave function. As an example we use the same two body potential used in the previous analysis and add the Coulomb potential:

$$
V(r)=-V_{0} \exp -\left(r / r_{0}\right)^{2}+\frac{e^{2}}{r} .
$$

For positive energies and $l=0$, the wave function behaves asymptotically as

$$
\Psi^{(c)}(r \rightarrow \infty)=A F_{c}(r)+B G_{c}(r),
$$

with $F_{c}(r), G_{c}(r)$ the regular and irregular Coulomb functions, respectively. The phase-shift is $\tan \delta_{c}=B / A$. The KVP remains valid when the long range Coulomb potential is considered and its form in terms of the integral relations results:

$$
\begin{gathered}
-\frac{m}{\hbar^{2}}<\Psi_{t}^{(c)}|H-E| F_{c}>=B \\
\frac{m}{\hbar^{2}}<\Psi_{t}^{(c)}|H-E| \tilde{G}_{c}>=A \\
{\left[\tan \delta_{c}\right]^{2^{n d}}=\frac{B}{A} .}
\end{gathered}
$$

with $\tilde{G}_{c}=f_{r e g} G_{c}$ and $\Psi_{t}^{(c)}$ a trial wave function behaving asymptotically as $\Psi^{(c)}$. Since $(H-E) \mid F_{c}>$ and $(H-$ $E) \mid \tilde{G}_{c}>$ go to zero outside the range of the short range potential, the integrals in Eq. (71) are negligible outside that region. Therefore, for the computation of the phase-shift it is enough to require that $\Psi_{t}^{(c)}$ verifies $(H-E) \Psi_{t}^{(c)}=0$, inside that region. To exploit this fact, we introduce the following screened potential:

$$
V_{s c}(r)=-V_{0} \exp \left[-\left(r / r_{0}\right)^{2}\right]+\left[\mathrm{e}^{-\left(r / r_{s c}\right)^{n}}\right] \frac{e^{2}}{r} .
$$

For specific values of $n$ and $r_{s c}$ it has the property of being extremely close to the potential $V(r)$ of Eq. (69) for $r<r_{0}$, with $r_{0}$ the range of the short range potential. The screening factor $\mathrm{e}^{-\left(r / r_{s c}\right)^{n}}$ cuts the Coulomb potential for $r>r_{s c}$. Using the potential $V_{s c}$ to describe a scattering process, the wave function behaves asymptotically as

$$
\Psi_{n, r_{s c}}(r \rightarrow \infty)=A F(r)+B G(r)
$$

with $F, G$ from Eq. (68), since $V_{s c}$ is a short range potential. Solving the Schrödinger equation for this potential, it is possible to obtain the wave function $\Psi_{n, r_{s c}}$ for different values of $n$ and $r_{s c}$. This wave function can be considered as a

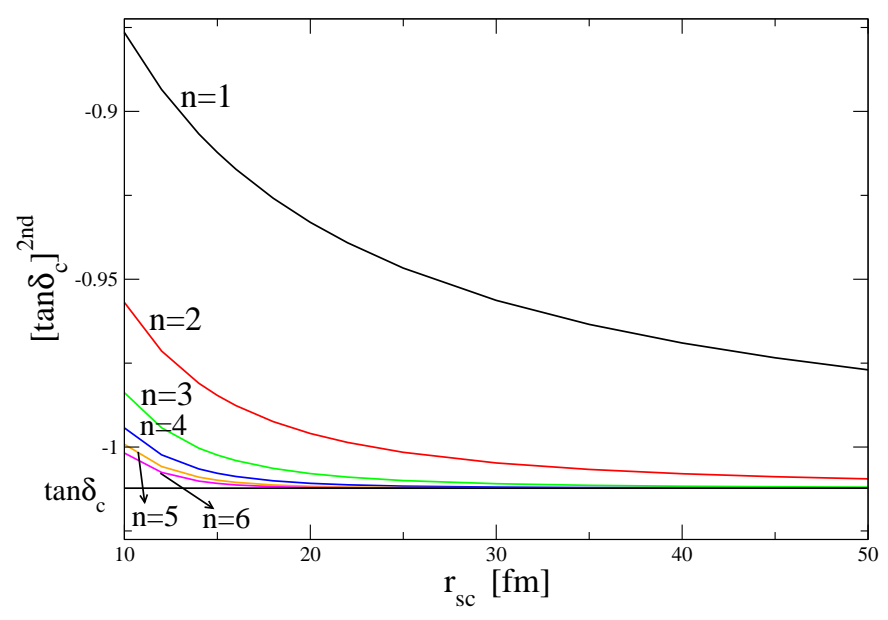

Fig. 8. The two-nucleon second order estimate $\left[\tan \delta_{c}\right]^{2^{n d}}$ as a function of $r_{s c}$ for different values of $n$. As a reference the exact value for $\tan \delta_{c}$ is given as a straight line.

trial wave function for the problem in which the Coulomb potential is unscreened. Accordingly it can be used as input in Eq. (71) to obtain a second order estimate of the Coulomb phase-shift,

$$
\begin{gathered}
-\frac{m}{\hbar^{2}}<\Psi_{n, r_{s c}}|H-E| F_{c}>=B \\
\frac{m}{\hbar^{2}}<\Psi_{n, r_{s c}}|H-E| \tilde{G}_{c}>=A \\
{\left[\tan \delta_{c}\right]^{2^{n d}}=\frac{B}{A}}
\end{gathered}
$$

where in $H$ the unscreened Coulomb potential is considered. This estimate depends on $n$ and $r_{s c}$ as the wave function does. In Fig. 8 the second order estimate $\left[\tan \delta_{c}\right]^{2^{n d}}$ is shown as a function of $r_{s c}$ for different values of $n$. The straight line is the exact value of $\tan \delta_{c}$ obtained solving the Schrödinger equation. We can observe that for $n \geq 4$ and $r_{s c}>30 \mathrm{fm}$ the second order estimate coincides with the exact results. In this example the integral relations derived from the Kohn Variational Principle have been used to extract a phase-shift in presence of the Coulomb potential using wave functions with free asymptotic conditions.

Finally an application of the integral relations to the $A=3$ system is discussed. To this end we give the generalization of the integral relations to the case in which more than one channel is open. The coefficients $A$ and $B$ of Eq. (68) correspond to matrices

$$
\begin{aligned}
B_{i j} & =-\frac{m}{\hbar^{2}}<\Psi_{i}|H-E| F_{j}> \\
A_{i j} & =\frac{m}{\hbar^{2}}<\Psi_{i}|H-E| \widetilde{G}_{j}> \\
R^{2^{n d}} & =A^{-1} B .
\end{aligned}
$$

with $R^{2^{n d}}$ the second order estimate of the scattering matrix whose eigenvalues are the phase shifts and the indices 
$19^{\text {th }}$ International IUPAP Conference on Few-Body Problems in Physics

$(i, j)$ indicate the different asymptotic configurations accessible at the specific energy under consideration. We consider $p-d$ scattering at $E_{l a b}=3 \mathrm{MeV}$ using the AV18 potential in the $J=1 / 2^{+}$state. The corresponding scattering matrix is a $2 \times 2$ matrix. The corresponding phaseshift and mixing parameters have been calculated using the PHH expansion and are given in Table 3. From the previous discussion we have shown that it is possible to solve an equivalent problem with a screened Coulomb potential, so with free asymptotic conditions, and then use the integral relations to extract the scattering matrix corresponding to the unscreened problem. This has been done using Eq. (75) and the results are given in Table 3 using $r_{s c}=50 \mathrm{fm}$ and $n_{s c}=5$. We observe a complete agreement between the two procedures.

Table 3. Phase-shift and mixing parameters for $p-d$ scattering at $E_{\text {lab }}=3 \mathrm{MeV}$ using the AV18 potential. Results using the $\mathrm{PHH}$ expansion (second column) and using the integral relations (last column)

\begin{tabular}{ccc}
\hline & $p-d$ & Int.Rel. \\
\hline${ }^{4} D_{1 / 2}$ & $-3.563^{\circ}$ & $-3.562^{\circ}$ \\
${ }^{2} S_{1 / 2}$ & $-32.12^{\circ}$ & $-32.12^{\circ}$ \\
$\eta_{1 / 2+}$ & $1.100^{\circ}$ & $1.101^{\circ}$ \\
\hline
\end{tabular}

\section{Conclusions}

Stimulated by the fact that the commonly used TNF models do not reproduce simultaneously the triton and ${ }^{4} \mathrm{He}$ binding energy and the $n-d$ doublet scattering length, we have analyzed possible modifications of some of the TNF models usually used in the description of light nuclei: the TM' and the URIX models. We have also considered the recent N2LOL model. In each of these models we have varied the original parameters so as to improve the description of the mentioned quantities. Furthermore we have studied the description of some $p-d$ polarization observables at $E_{l a b}=3 \mathrm{MeV}$. We have observed that the modification of the URIX produces a worse description of the vector polarization observables due to the artificial increase of the strength of the repulsive term. The analysis of the TM' model has put in evidence the necessity of including a repulsive term. In the case of the N2LOL model a fine tuning of the parameters was possible in order to have an acceptable description of the triton and ${ }^{4} \mathrm{He}$ binding energies and the $n-d$ doublet scattering length. Moreover, in the polarization observables we observe an improvement in the vector analyzing powers and a slightly worse description of $T_{21}$. From this analysis we have established a connection between the short-range structure of the TNF and the polarization observables at low energies.

In a different application, we have discussed the use of the integral relations derived from the KVP in the description of scattering states. Firstly we have shown the use of bound state like wave functions to compute the scattering matrix and, in the case of charged particles, the possibility of computing phase-shifts using scattering wave functions with free asymptotic conditions, obtained after screening the Coulomb interaction. Both problems are of interest in the study of light nuclei.

\section{Acknowledgments}

This work has been done in collaboration with my colleagues in Pisa M. Viviani, L. Girlanda and L.E. Marcucci, with C. Romero-Redondo and E. Garrido (CSIC) and P. Barletta (UCL).

\section{References}

1. W. Glöckle, H. Witała, D. Hüber, H. Kamada, and J. Golak, Phys. Rep. 274 (1994) 107

2. A. Kievsky, M. Viviani, and S. Rosati, Phys. Rev. C64 (2001) 024002

3. S. Coon and W. Glöckle, Phys. Rev. C23 (1981) 1790

4. B.S. Pudliner et al., Phys. Rev. Lett. 51 (1995) 4396

5. J.L. Friar, D. Hüber, and U. van Kolck, Phys. Rev. C59 (1999) 53

6. S.A. Coon and H.K. Han, Few-Body Syst. 30 (2001) 131

7. H.T. Coelho, T.K. Das, and M.R. Robilotta, Phys. Rev. C28 (1983) 1812; M.R. Robilotta and H.T. Coelho, Nucl. Phys. A460, (1986) 645

8. E. Epelbaum et al., Phys. Rev. C66 (2002) 064001

9. P. Navrátil, Few-Body Syst. 41 (2007) 117

10. S.K. Bogner et al., Nucl. Phys. A784, (2007) 79

11. A.C. Phillips, Nucl. Phys. A107, (1968) 209

12. P.F. Bedaque, H.-W. Hammer, and U. van Kolck, Nucl. Phys. A646, (1999) 444

13. A. Kievsky, S. Rosati, M. Viviani, L.E. Marcucci, and L. Girlanda, J. Phys. G: Nucl. Part. Phys. 35 (2008) 063101

14. L.E. Marcucci, A. Kievsky, L. Girlanda, S. Rosati and M. Viviani, Phys. Rev. C80 (2009) 034003

15. R.B. Wiringa, V.G.J. Stoks, and R. Schiavilla, Phys. Rev. C51 (1995) 38

16. D.R. Entem and R. Machleidt, Phys. Rev. C68 (2003) 041001

17. A. Kievsky, M. Viviani, and S. Rosati, Nucl. Phys. A577, (1994) 511

18. A. Kievsky, Nucl. Phys. A624, (1997) 125

19. M. Viviani, A. Kievsky, and S. Rosati, Phys. Rev. C71 (2005) 024006

20. R. Lazauskas et al., Phys.Rev. C71 (2005) 034004

21. M. Viviani, L.E. Marcucci, S. Rosati, A. Kievsky, and L. Girlanda; Few-Body Syst.39 (2006) 159

22. P. Barletta, C. Romero-Redondo, A. Kievsky, M. Viviani and E. Garrido, Phys.Rev.Lett. 103 (2009) 090402

23. F.E. Harris, Phys.Rev.Lett. 19 (1967) 173

24. A.S. Kadyrov, I. Bray, A.M. Mukhamedzhanov, and A.T. Stelbovics, Annals of Phys. 324 (2009) 1516

25. J. Raynal and J. Revai, Il Nuovo Cim. 68 (1970) 612 
EPJ Web of Conferences

26. C.R. Chen et al., Phys. Rev. C39 (1989) 1261

27. K. Schoen et al., Phys. Rev. C67 (2003) 044005

28. A. Nogga, H. Kamada, W. Glöckle, and B.R. Barrett, Phys. Rev. C65 (2002) 054002

29. S. Shimizu et al., Phys. Rev. C52 (1995) 1193 